Article

\title{
Recursive Algorithms for Multivariable Output-Error-Like ARMA Systems
}

\author{
Hao Ma ${ }^{1}{ }^{1}$, Jian Pan ${ }^{1, *}$, Lei Lv ${ }^{1}$, Guanghui Xu ${ }^{1}$, Feng Ding ${ }^{2,3, *} \mathbb{C}$, Ahmed Alsaedi ${ }^{4}$ and \\ Tasawar Hayat 4 \\ 1 Hubei Key Laboratory for High-efficiency Utilization of Solar Energy and Operation Control of Energy \\ Storage System, School of Electrical and Electronic Engineering, Hubei University of Technology, Wuhan \\ 430068, China; haomawoshi@126.com (H.M.); lvlei9076@163.com (L.L.); xuguanghui29@163.com (G.X.) \\ 2 College of Automation and Electronic Engineering, Qingdao University of Science and Technology, \\ Qingdao 266061, China \\ 3 School of Internet of Things Engineering, Jiangnan University, Wuxi 214122, China \\ 4 Department of Mathematics, King Abdulaziz University, Jeddah 21589, Saudi Arabia; \\ aalsaedi@hotmail.com (A.A.); fmgpak@gmail.com (T.H.) \\ * Correspondence: jpan@163.com (J.P.); fdingab@126.com (F.D.)
}

Received: 26 May 2019; Accepted: 12 June 2019; Published: 19 June 2019

\begin{abstract}
This paper studies the parameter identification problems for multivariable output-error-like systems with colored noises. Based on the hierarchical identification principle, the original system is decomposed into several subsystems. However, each subsystem contains the same parameter vector, which leads to redundant computation. By taking the average of the parameter estimation vectors of each subsystem, a partially-coupled subsystem recursive generalized extended least squares (PC-S-RGELS) algorithm is presented to cut down the redundant parameter estimates. Furthermore, a partially-coupled recursive generalized extended least squares (PC-RGELS) algorithm is presented to further reduce the computational cost and the redundant estimates by using the coupling identification concept. Finally, an example indicates the effectiveness of the derived algorithms.
\end{abstract}

Keywords: system identification; recursive algorithm; least squares; multivariable system; coupling identification concept

\section{Introduction}

System identification is an important branch in the field of modern control and is an important method to establish systematic mathematical models from the combination of observation data and prior knowledge [1-8], and has been applied in many fields for decades, such as controller design [9-15] and system analysis [16-20]. Parameter identification is an important part of system identification and is to estimate the parameters by using the measurable data [21-27]. Parameter estimation methods can be applied to many areas [28-31]. Recently, in the literature of parameter identification, Wan et al. studied the problem of parameter estimation for multivariable equation-error systems with colored noises and proposed a hierarchical gradient-based iterative identification algorithm by using the hierarchical identification principle [32]. Chen et al. transformed the time-delay rational model into an augmented model based on the redundant rule and proposed a biased compensation recursive least squares-based threshold algorithm [33]. Other identification methods can be found in [34-40].

Multivariable systems widely consist in practical industrial processes on account of multi-input multi-output systems can describe modern industrial process more accurately [41-45]. Parameter estimation of multivariable systems has attracted extensive research attention over the past decades, and many different identification approaches have been proposed to solve the parameter identification 
problems of multivariable systems, such as the hierarchical identification principle and the coupling identification concept [46,47]. The core idea of the hierarchical identification principle is to decompose the original model into several submodels, and to combine other approaches to estimate the parameters of the submodels $[48,49]$. The coupled identification methods have been derived to identify the parameters of multivariable systems and were first presented in [50]. The basic idea of the coupling identification concept is to decompose the original system into several subsystems, and to estimate the parameters based on the coupled parameter relationships between these subsystems [51-53].

In the field of system modeling and control, the recursive identification and iterative identification methods are basic [54-59]. The recursive least squares methods are the commonly used parameter estimation approaches among many different parameter estimation techniques [60-62]. Recently, the recursive least squares (RLS) algorithm is always combined other methods to identify the complex systems. For instance, Zhang et al. proposed a filtering-based two-stage RLS algorithm for a bilinear system which is described by the state space form based on the filtering technique [63]. Liu et al. sudied the parameter estimation problems of multivariate output-error autoregressive systems and derived a filtering-based auxiliary model recursive generalized least squares algorithm based on the data filtering technique and the auxiliary model identification idea [64].

Multivariable output-error-like systems are special type of multivariable systems, which contain not only multiple inputs and multiple outputs, but also more complex parameter forms, i.e., the parameter vector and the parameter matrix $[65,66]$. Hence, the multivariable output-error like systems can describe modern industrial process more accurately than other types of multivariable systems. Recently, for multivariate output-error systems, Wang et al. proposed a decomposition based recursive least squares identification algorithm by using the auxiliary model, and analyzed its convergence through the stochastic process theory [67]. Ding proposed a hierarchical iterative identification algorithm for multi-input output-error systems with autoregressive noise [68]. Different from the methods in $[67,68]$, this paper studies the parameter identification problems of multivariable output-error-like (M-OE-like) systems with colored noises which is described by the autoregressive moving average (ARMA) model by means of the decomposition technique and the coupling identification concept $[69,70]$. The main contributions of this paper lie in the following aspects.

- Based on the hierarchical identification principle, this paper decomposes the original system into $m$ subsystems.

- Based on the coupled relationships between subsystems, a partially-coupled recursive generalized extended least squares (PC-RGELS) algorithm is proposed to identify the parameters of M-OEARMA-like systems.

- The derived PC-RGELS algorithm has higher computation efficiency and higher estimation accuracy than the recursive generalized extended least squares (RGELS) algorithm.

This paper is organized as follows. Section 2 describes the identification model. A RGELS algorithm is proposed to give some comparisons with the proposed algorithms in Section 3. Section 4 proposes a partially-coupled recursive generalized extended least squares algorithm. Section 5 provides the numerical simulation results to illustrate the performance of the proposed algorithms. Finally, Section 6 gives some conclusions.

\section{The System Description}

Let us introduce some symbols. " $B=: Y$ " or " $Y:=B$ " stands for " $B$ is defined as $Y$ "; the superscript T stands for the vector/matrix transpose; the symbol $\boldsymbol{I}_{n}$ denotes an identity matrix of size $n \times n ; \mathbf{1}_{n}$ stands for an $n$-dimensional column vector whose elements are 1 ; the symbol $\otimes$ represents the Kronecker product, for example, $\boldsymbol{C}=\left[c_{i j}\right] \in \mathbb{R}^{m \times n}, \boldsymbol{D}=\left[d_{i j}\right] \in \mathbb{R}^{p \times q}, \boldsymbol{C} \otimes \boldsymbol{D}=\left[c_{i j} \boldsymbol{D}\right] \in \mathbb{R}^{(m p) \times(n q)}$, in general $\boldsymbol{C} \otimes \boldsymbol{D} \neq \boldsymbol{D} \otimes \boldsymbol{C} ; \operatorname{col}[\boldsymbol{Y}]$ is defined as the vector formed by all columns of matrix $\boldsymbol{Y}$ arranged in order, for example, $\boldsymbol{Y}=\left[\boldsymbol{y}_{1}, \boldsymbol{y}_{2}, \cdots, \boldsymbol{y}_{n}\right] \in \mathbb{R}^{m \times n}, \operatorname{col}[\boldsymbol{Y}]=\left[\boldsymbol{y}_{1}^{\mathrm{T}}, \boldsymbol{y}_{2}^{\mathrm{T}}, \cdots, \boldsymbol{y}_{n}^{\mathrm{T}}\right]^{\mathrm{T}} \in \mathbb{R}^{m n} ; \hat{\boldsymbol{Y}}(s)$ denotes the estimate of $\boldsymbol{Y}$ at time $s$; the norm of a matrix (or a column vector) $\boldsymbol{Y}$ is defined by $\|\boldsymbol{Y}\|^{2}:=\operatorname{tr}\left[\boldsymbol{Y} \boldsymbol{Y}^{\mathrm{T}}\right]$. 
Recently, for the multivariable output-error system:

$$
\boldsymbol{y}(s)=\frac{\boldsymbol{\Phi}_{s}(s) \boldsymbol{\theta}}{A(z)}+\boldsymbol{v}(s)
$$

where $\boldsymbol{y}(s):=\left[y_{1}(s), y_{2}(s), \cdots, y_{m}(s)\right]^{\mathrm{T}} \in \mathbb{R}^{m}$ refers to the output vector of the system, $\boldsymbol{v}(s) \in \mathbb{R}^{m}$ is the white noise vector with zero mean, $\boldsymbol{\Phi}_{s}(s) \in \mathbb{R}^{m \times n}$ is the information matrix consisting of the input-output data, $\boldsymbol{\theta} \in \mathbb{R}^{n}$ is the parameter vector, $z^{-1}$ is a unit delay operator: $z^{-1} y(s)=y(s-1)$, $A(z)$ is a monic polynomial in $z^{-1}$, and

$$
A(z):=1+a_{1} z^{-1}+a_{2} z^{-2}+\cdots+a_{n_{a}} z^{n_{a}}, \quad a_{i} \in \mathbb{R},
$$

a coupled stochastic gradient identification algorithm has been proposed to estimate the parameters of this system [71].

Different from the system in [71], this paper considers the multivariable output-error-like system with autoregressive moving average noise:

$$
\boldsymbol{y}(s)=\frac{Q(z)}{\alpha(z)} \boldsymbol{u}(s)+\frac{D(z)}{C(z)} \boldsymbol{v}(s),
$$

where the definitions of $\boldsymbol{y}(s)$ and $\boldsymbol{v}(s)$ are same to above, $\boldsymbol{u}(s):=\left[u_{1}(s), u_{2}(s), \cdots, u_{r}(s)\right]^{\mathrm{T}} \in \mathbb{R}^{r}$ is the system input vector, $\alpha(z), C(z)$ and $D(z)$ are monic polynomials in $z^{-1}$ and $Q(z)$ is a matrix-coefficient polynomial in $z^{-1}$, and defined by

$$
\begin{aligned}
& \alpha(z):=1+a_{1} z^{-1}+a_{2} z^{-2}+\cdots+a_{n} z^{-n}, \quad a_{i} \in \mathbb{R}, \\
& Q(z):=Q_{1} z^{-1}+Q_{2} z^{-2}+\cdots+Q_{n} z^{-n}, \quad Q_{i} \in \mathbb{R}^{m \times r}, \\
& C(z):=1+c_{1} z^{-1}+c_{2} z^{-2}+\cdots+c_{n_{c}} z^{n_{c}}, \quad c_{i} \in \mathbb{R}, \\
& D(z):=1+d_{1} z^{-1}+d_{2} z^{-2}+\cdots+d_{n_{d}} z^{n_{d}}, \quad d_{i} \in \mathbb{R} .
\end{aligned}
$$

In order to focus on the essence of the parameter estimation, we assume that the orders $m, r, n, n_{c}$ and $n_{d}$ are known, and $\boldsymbol{y}(s)=\mathbf{0}, \boldsymbol{u}(s)=\mathbf{0}$ and $\boldsymbol{v}(s)=\mathbf{0}$ for $s \leqslant 0$. Define the actual output vector of the system,

$$
x(s):=\frac{Q(z)}{\alpha(z)} \boldsymbol{u}(s) \in \mathbb{R}^{m} .
$$

Define the parameter vector $\alpha$ and the parameter matrix $\theta$, and the information vector $\varphi(s)$ and the information matrix $\psi_{s}(s)$ as

$$
\begin{aligned}
\boldsymbol{\alpha} & :=\left[a_{1}, a_{2}, \cdots, a_{n}\right]^{\mathrm{T}} \in \mathbb{R}^{n}, \\
\boldsymbol{\theta}^{\mathrm{T}} & :=\left[\boldsymbol{Q}_{1}, \boldsymbol{Q}_{2}, \cdots, \boldsymbol{Q}_{n}\right] \in \mathbb{R}^{m \times(r n)}, \\
\boldsymbol{\varphi}(s) & :=\left[\boldsymbol{u}^{\mathrm{T}}(s-1), \boldsymbol{u}^{\mathrm{T}}(s-2), \cdots, \boldsymbol{u}^{\mathrm{T}}(s-n)\right]^{\mathrm{T}} \in \mathbb{R}^{r n}, \\
\boldsymbol{\psi}_{s}(s) & :=[-\boldsymbol{x}(s-1),-\boldsymbol{x}(s-2), \cdots,-\boldsymbol{x}(s-n)] \in \mathbb{R}^{m \times n} .
\end{aligned}
$$

Equation (3) can be expressed as

$$
\begin{aligned}
\boldsymbol{x}(s) & =[1-\alpha(z)] \boldsymbol{x}(s)+\boldsymbol{Q}(z) \boldsymbol{u}(s), \\
& =\boldsymbol{\psi}_{s}(s) \boldsymbol{\alpha}+\boldsymbol{\theta}^{\mathrm{T}} \boldsymbol{\varphi}(s) .
\end{aligned}
$$

Define an internal vector of the system,

$$
\boldsymbol{w}(s):=\frac{D(z)}{C(z)} \boldsymbol{v}(s) \in \mathbb{R}^{m} .
$$


Let $n_{1}:=n_{c}+n_{d}$, define the parameter vector $\rho$ and the information matrix $\psi_{n}(s)$ of the system as

$$
\rho:=\left[c_{1}, c_{2}, \cdots, c_{n_{c}}, d_{1}, d_{2}, \cdots, d_{n_{d}}\right]^{\mathrm{T}} \in \mathbb{R}^{n_{1}},
$$

$\boldsymbol{\psi}_{n}(s):=\left[-\boldsymbol{w}(s-1),-\boldsymbol{w}(s-2), \cdots,-\boldsymbol{w}\left(s-n_{\mathcal{c}}\right), \boldsymbol{v}(s-1), \boldsymbol{v}(s-2), \cdots, \boldsymbol{v}\left(s-n_{d}\right)\right] \in \mathbb{R}^{m \times n_{1}}$.

Equation (5) can be rewritten as

$$
\begin{aligned}
\boldsymbol{w}(s) & =[1-C(z)] \boldsymbol{w}(s)+D(z) \boldsymbol{v}(s), \\
& =\boldsymbol{y}(s)-\boldsymbol{\psi}_{s}(s) \boldsymbol{\alpha}-\boldsymbol{\theta}^{\mathrm{T}} \boldsymbol{\varphi}(s), \\
& =\boldsymbol{\psi}_{n}(s) \boldsymbol{\rho}+\boldsymbol{v}(s) .
\end{aligned}
$$

Using (4) and (7), Equation (2) can be equivalently written as

$$
\begin{aligned}
\boldsymbol{y}(s) & =\boldsymbol{x}(s)+\boldsymbol{w}(s), \\
& =\boldsymbol{\psi}_{s}(s) \boldsymbol{\alpha}+\boldsymbol{\theta}^{\mathrm{T}} \boldsymbol{\varphi}(s)+\boldsymbol{\psi}_{n}(s) \boldsymbol{\rho}+\boldsymbol{v}(s), \\
& =\left[\boldsymbol{\psi}_{s}(s), \boldsymbol{\psi}_{n}(s)\right]\left[\begin{array}{l}
\boldsymbol{\alpha} \\
\boldsymbol{\rho}
\end{array}\right]+\boldsymbol{\theta}^{\mathrm{T}} \boldsymbol{\varphi}(s)+\boldsymbol{v}(s) .
\end{aligned}
$$

Let $n_{0}:=n_{1}+n$, define the information matrix $\psi(s)$ and the parameter vector $\beta$ as

$$
\begin{aligned}
\psi(s) & :=\left[\boldsymbol{\psi}_{s}(s), \boldsymbol{\psi}_{n}(s)\right] \in \mathbb{R}^{m \times n_{0}}, \\
\boldsymbol{\beta} & :=\left[\boldsymbol{\alpha}^{\mathrm{T}}, \boldsymbol{\rho}^{\mathrm{T}}\right]^{\mathrm{T}} \in \mathbb{R}^{n_{0}} .
\end{aligned}
$$

Substituting (10) and (11) into (9) gives the hierarchical identification model

$$
\boldsymbol{y}(s)=\boldsymbol{\psi}(s) \boldsymbol{\beta}+\boldsymbol{\theta}^{\mathrm{T}} \boldsymbol{\varphi}(s)+\boldsymbol{v}(s) .
$$

For convenience, we define an information matrix $\Psi(s)$ by making use of the Kronecker product of the information matrix $\psi(s)$ and the information vector $\varphi(s)$ as

$$
\boldsymbol{\Psi}(s):=\left[\boldsymbol{\psi}(s), \boldsymbol{\varphi}^{\mathrm{T}}(s) \otimes \boldsymbol{I}_{m}\right] \in \mathbb{R}^{m \times n_{2}}, \quad n_{2}:=n_{0}+m r n .
$$

Hence, a parameter vector $\vartheta$ is defined by using the parameter vector $\beta$ and the parameter matrix $\theta$,

$$
\vartheta:=\left[\begin{array}{c}
\beta \\
\operatorname{col}\left[\boldsymbol{\theta}^{\mathrm{T}}\right]
\end{array}\right] \in \mathbb{R}^{n_{2}} .
$$

Then Equation (12) can be equivalently expressed as

$$
\boldsymbol{y}(s)=\boldsymbol{\Psi}(s) \boldsymbol{\vartheta}+\boldsymbol{v}(s) .
$$

Therefore, we get the identification model (13) of the M-OEARMA-like system in (2), where $\vartheta$ is the parameter vector to be identified and contains all the parameters of the system (2)

\section{The RGELS Algorithm}

Based on Equation (13), define a criterion function,

$$
J_{1}(\boldsymbol{\vartheta}):=\sum_{j=1}^{s}\|\boldsymbol{y}(j)-\boldsymbol{\Psi}(j) \boldsymbol{\vartheta}\|^{2} .
$$


Let $\hat{\boldsymbol{\vartheta}}(s)$ be the estimate of $\boldsymbol{\vartheta}$ at time $s$. Minimizing $J_{1}(\boldsymbol{\vartheta})$ gives

$$
\left.\frac{\partial J_{1}(\boldsymbol{\vartheta})}{\partial \boldsymbol{\vartheta}}\right|_{\vartheta=\hat{\boldsymbol{\vartheta}}(s)}=\left.\frac{\partial\left(\boldsymbol{Y}_{S}-\boldsymbol{H}_{s} \boldsymbol{\vartheta}\right)^{\mathrm{T}}\left(\boldsymbol{Y}_{S}-\boldsymbol{H}_{s} \boldsymbol{\vartheta}\right)}{\partial \boldsymbol{\vartheta}}\right|_{\boldsymbol{\vartheta}=\hat{\boldsymbol{\vartheta}}(s)}=\mathbf{0},
$$

where

$$
\begin{aligned}
\boldsymbol{Y}_{s} & :=\left[\boldsymbol{y}^{\mathrm{T}}(1), \boldsymbol{y}^{\mathrm{T}}(2), \boldsymbol{y}^{\mathrm{T}}(3), \cdots, \boldsymbol{y}^{\mathrm{T}}(s)\right]^{\mathrm{T}} \in \mathbb{R}^{m s}, \\
\boldsymbol{H}_{s} & :=\left[\boldsymbol{\Psi}^{\mathrm{T}}(1), \boldsymbol{\Psi}^{\mathrm{T}}(2), \boldsymbol{\Psi}^{\mathrm{T}}(3), \cdots, \boldsymbol{\Psi}^{\mathrm{T}}(s)\right]^{\mathrm{T}} \in \mathbb{R}^{(m s) \times n_{2},}
\end{aligned}
$$

Parameter estimate $\hat{\boldsymbol{\vartheta}}(s)$ of $\vartheta$ can be obtained from (15) as

$$
\begin{aligned}
\hat{\boldsymbol{\vartheta}}(s) & =\left(\boldsymbol{H}_{s}^{\mathrm{T}} \boldsymbol{H}_{s}\right)^{-1} \boldsymbol{H}_{s}^{\mathrm{T}} \boldsymbol{Y}_{s}, \\
& =\left[\sum_{j=1}^{s} \boldsymbol{\Psi}^{\mathrm{T}}(j) \boldsymbol{\Psi}(j)\right]^{-1}\left[\sum_{j=1}^{s} \boldsymbol{\Psi}^{\mathrm{T}}(j) \boldsymbol{y}(j)\right] .
\end{aligned}
$$

Define the covariance matrix

$$
\begin{aligned}
\boldsymbol{P}^{-1}(s) & :=\sum_{j=1}^{s} \boldsymbol{\Psi}^{\mathrm{T}}(j) \boldsymbol{\Psi}(j) \in \mathbb{R}^{n_{2} \times n_{2}}, \\
& =\boldsymbol{P}^{-1}(s-1)+\boldsymbol{\Psi}^{\mathrm{T}}(s) \boldsymbol{\Psi}(s) .
\end{aligned}
$$

Let $\boldsymbol{L}(s):=\boldsymbol{P}(s) \boldsymbol{\Psi}^{\mathrm{T}}(s) \in \mathbb{R}^{n_{2} \times m}$ be the gain matrix. Based on the derivation of the RLS algorithm in $[72,73]$, we can easily get the RLS relations:

$$
\begin{aligned}
\hat{\boldsymbol{\vartheta}}(s) & =\hat{\boldsymbol{\vartheta}}(s-1)+\boldsymbol{L}(s)[\boldsymbol{y}(s)-\boldsymbol{\Psi}(s) \hat{\boldsymbol{\vartheta}}(s-1)], \\
\boldsymbol{L}(s) & =\boldsymbol{P}(s-1) \boldsymbol{\Psi}^{\mathrm{T}}(s)\left[\boldsymbol{I}_{m}+\boldsymbol{\Psi}(s) \boldsymbol{P}(s-1) \boldsymbol{\Psi}^{\mathrm{T}}(s)\right]^{-1}, \\
\boldsymbol{P}(s) & =\boldsymbol{P}(s-1)-\boldsymbol{L}(s)\left[\boldsymbol{P}(s-1) \boldsymbol{\Psi}^{\mathrm{T}}(s)\right]^{\mathrm{T}} .
\end{aligned}
$$

However, Equations (19)-(21) cannot figure out the parameter estimate $\hat{\boldsymbol{\vartheta}}(s)$ because of the information matrix $\boldsymbol{\Psi}(s)$ contains the unknown vectors $\boldsymbol{x}(s-i), \boldsymbol{w}(s-i)$ and $\boldsymbol{v}(s-i)$. The solution is to replace these unknown vectors in $\Psi(s)$ with their corresponding estimates $\hat{x}(s-i), \hat{w}(s-i)$ and $\hat{v}(s-i)$ by using the auxiliary model. Define the estimates of $\boldsymbol{\Psi}(s), \psi(s), \psi_{s}(s)$ and $\psi_{n}(s)$ as

$$
\begin{aligned}
\hat{\boldsymbol{\Psi}}(s) & :=\left[\hat{\boldsymbol{\psi}}(s), \boldsymbol{\varphi}^{\mathrm{T}}(s) \otimes \boldsymbol{I}_{m}\right] \in \mathbb{R}^{m \times n_{2}} \\
\hat{\boldsymbol{\psi}}(s) & :=\left[\hat{\boldsymbol{\psi}}_{s}(s), \hat{\boldsymbol{\psi}}_{n}(s)\right] \in \mathbb{R}^{m \times n_{0}}, \\
\hat{\boldsymbol{\psi}}_{s}(s) & :=[-\hat{\boldsymbol{x}}(s-1),-\hat{\boldsymbol{x}}(s-2), \cdots,-\hat{\boldsymbol{x}}(s-n)] \in \mathbb{R}^{m \times n}, \\
\hat{\boldsymbol{\psi}}_{n}(s) & :=\left[-\hat{\boldsymbol{w}}(s-1), \cdots,-\hat{\boldsymbol{w}}\left(s-n_{c}\right), \hat{\boldsymbol{v}}(s-1), \cdots, \hat{\boldsymbol{v}}\left(s-n_{d}\right)\right] \in \mathbb{R}^{m \times n_{1}}
\end{aligned}
$$

Replacing $\boldsymbol{\psi}_{s}(s), \boldsymbol{\alpha}$ and $\boldsymbol{\theta}$ in (4) and (6) with their estimates $\hat{\boldsymbol{\psi}}_{s}(s), \hat{\boldsymbol{\alpha}}(s)$ and $\hat{\boldsymbol{\theta}}(s)$, the estimates $\hat{\boldsymbol{x}}(s)$ and $\hat{\boldsymbol{w}}(s)$ can be calculated by two auxiliary models:

$$
\begin{aligned}
\hat{\boldsymbol{x}}(s) & :=\hat{\boldsymbol{\psi}}_{s}(s) \hat{\boldsymbol{\alpha}}(s)+\hat{\boldsymbol{\theta}}^{\mathrm{T}}(s) \boldsymbol{\varphi}(s) \\
\hat{\boldsymbol{w}}(s) & :=\boldsymbol{y}(s)-\hat{\boldsymbol{x}}(s)=\boldsymbol{y}(s)-\hat{\boldsymbol{\psi}}_{s}(s) \hat{\boldsymbol{\alpha}}(s)-\hat{\boldsymbol{\theta}}^{\mathrm{T}}(s) \boldsymbol{\varphi}(s) .
\end{aligned}
$$

From (13), use the estimates $\hat{\boldsymbol{\Psi}}(s)$ and $\hat{\boldsymbol{\vartheta}}(s)$ of $\boldsymbol{\Psi}(s)$ and $\boldsymbol{\vartheta}$ to define the estimate of $\boldsymbol{v}(s)$ as

$$
\hat{\boldsymbol{v}}(s):=\boldsymbol{y}(s)-\hat{\mathbf{\Psi}}(s) \hat{\boldsymbol{\vartheta}}(s) .
$$

Combining (22)-(28) and replacing $\Psi(s)$ in (19)-(21) with $\hat{\Psi}(s)$ yield the following recursive generalized extended least squares (RGELS) algorithm: 


$$
\begin{aligned}
& \hat{\boldsymbol{\vartheta}}(s)=\hat{\boldsymbol{\vartheta}}(s-1)+\boldsymbol{L}(s)[\boldsymbol{y}(s)-\hat{\boldsymbol{\Psi}}(s) \hat{\boldsymbol{\vartheta}}(s-1)], \\
& \boldsymbol{L}(s)=\boldsymbol{P}(s-1) \hat{\boldsymbol{\Psi}}^{\mathrm{T}}(s)\left[\boldsymbol{I}_{m}+\hat{\boldsymbol{\Psi}}(s) \boldsymbol{P}(s-1) \hat{\boldsymbol{\Psi}}^{\mathrm{T}}(s)\right]^{-1}, \\
& \boldsymbol{P}(s)=\boldsymbol{P}(s-1)-\boldsymbol{L}(s)\left[\boldsymbol{P}(s-1) \hat{\boldsymbol{\Psi}}^{\mathrm{T}}(s)\right]^{\mathrm{T}}, \\
& \hat{\mathbf{\Psi}}(s)=\left[\hat{\boldsymbol{\psi}}(s), \boldsymbol{\varphi}^{\mathrm{T}}(s) \otimes \boldsymbol{I}_{m}\right], \\
& \boldsymbol{\varphi}(s)=\left[\boldsymbol{u}^{\mathrm{T}}(s-1), \boldsymbol{u}^{\mathrm{T}}(s-2), \cdots, \boldsymbol{u}^{\mathrm{T}}(s-n)\right]^{\mathrm{T}}, \\
& \hat{\psi}(s)=\left[\hat{\psi}_{s}(s), \hat{\psi}_{n}(s)\right] \text {, } \\
& \hat{\psi}_{s}(s)=[-\hat{x}(s-1),-\hat{x}(s-2), \cdots,-\hat{x}(s-n)], \\
& \hat{\psi}_{n}(s)=\left[-\hat{\boldsymbol{w}}(s-1),-\hat{\boldsymbol{w}}(s-2), \cdots,-\hat{\boldsymbol{w}}\left(s-n_{c}\right), \hat{\boldsymbol{v}}(s-1), \hat{\boldsymbol{v}}(s-2), \cdots, \hat{\boldsymbol{v}}\left(s-n_{d}\right)\right], \\
& \hat{\boldsymbol{x}}(s)=\hat{\boldsymbol{\psi}}_{s}(s) \hat{\boldsymbol{\alpha}}(s)+\hat{\boldsymbol{\theta}}^{\mathrm{T}}(s) \boldsymbol{\varphi}(s), \\
& \hat{\boldsymbol{w}}(s)=\boldsymbol{y}(s)-\hat{\boldsymbol{\psi}}_{s}(s) \hat{\boldsymbol{\alpha}}(s)-\hat{\boldsymbol{\theta}}^{\mathrm{T}}(s) \boldsymbol{\varphi}(s), \\
& \hat{\boldsymbol{v}}(s)=\boldsymbol{y}(s)-\hat{\boldsymbol{\Psi}}(s) \hat{\boldsymbol{v}}(s) \text {. }
\end{aligned}
$$

The procedure contained in the RGELS algorithm in (29)-(39) as follows.

1. For $s \leqslant 0$, all variables are set to zero. Set the data length $L$. Let $s=1$, set the initial values $\hat{\boldsymbol{\vartheta}}(0)=\mathbf{1}_{n_{2}} / p_{0}, \hat{\boldsymbol{x}}(0)=\mathbf{1}_{m} / p_{0}, \hat{\boldsymbol{v}}(0)=\mathbf{1}_{m} / p_{0}, \hat{\boldsymbol{v}}(0)=\mathbf{1}_{m} / p_{0}, \boldsymbol{P}(0)=p_{0} \boldsymbol{I}_{n_{3}}, p_{0}=10^{6}$.

2. Collect the input-output data $\boldsymbol{u}(s)$ and $\boldsymbol{y}(s)$, and construct $\boldsymbol{\varphi}(s)$ using (33).

3. Form $\hat{\psi}_{s}(s), \hat{\psi}_{n}(s)$ and $\hat{\psi}(s)$ using (35)-(36) and (34), and form $\hat{\boldsymbol{\Psi}}(s)$ using (32).

4. Calculate the covariance matrix $\boldsymbol{P}(s)$ and the gain matrix $L(s)$ using (31) and (30), and update the estimate $\hat{\boldsymbol{\vartheta}}(s)$ using (29).

5. Figure the estimates $\hat{x}(s), \hat{\boldsymbol{w}}(s)$ and $\hat{\boldsymbol{v}}(s)$ using (37)-(39).

6. Compare $s$ with $L$ : if $s \leqslant L$, increase $s$ by 1 and go to Step 2; otherwise obtain the parameter estimate $\hat{\vartheta}(L)$ of $\vartheta$ and break up the program.

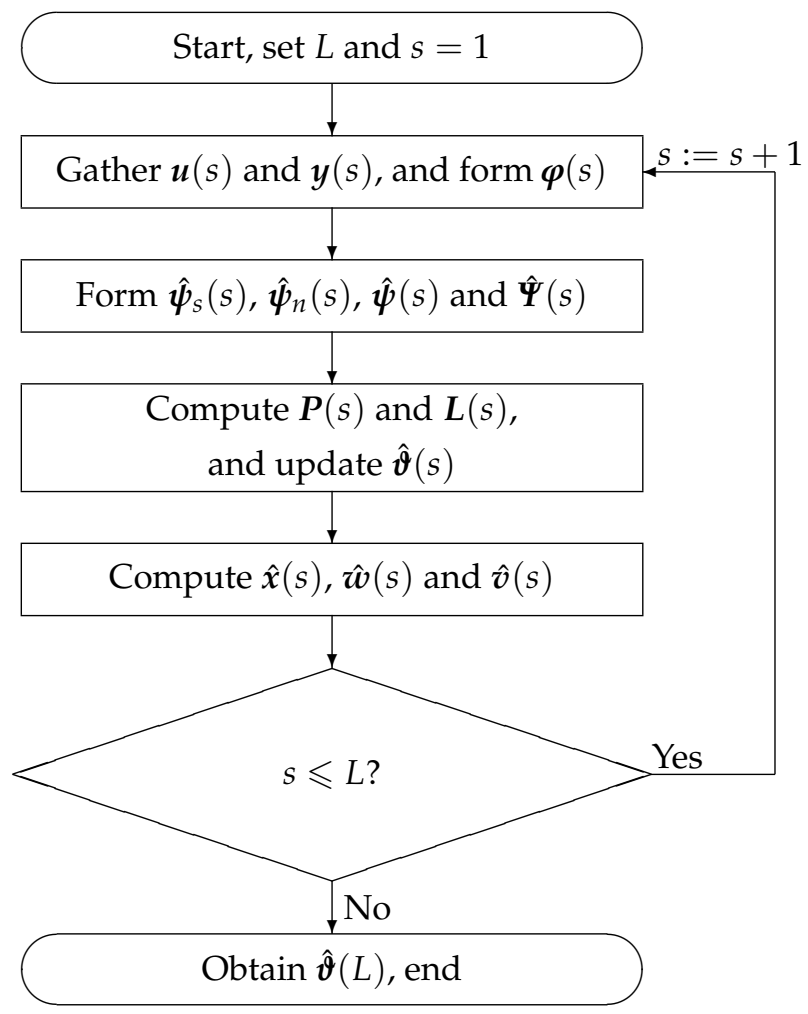

Figure 1. The flowchart of computing the RGELS parameter estimate $\hat{\vartheta}(L)$. 
The flowchart of computing $\hat{\boldsymbol{\vartheta}}(L)$ in the RGELS algorithm is shown in Figure 1. The RGELS algorithm is basic in system identification, and can be extended to study the parameter estimation problems of different systems such as signal modeling and communication networked systems [74-79].

\section{The PC-RGELS Algorithm}

In this part, a partially-coupled recursive generalized extended least squares (PC-RGELS) identification algorithm is studied to cut down the redundant estimates and improve the computational efficiency of the RGELS algorithm based on the decomposition technique and the coupling identification concept.

The identification model in (12) of system (2) is rewritten as follows:

$$
\boldsymbol{y}(s)=\psi(s) \boldsymbol{\beta}+\boldsymbol{\theta}^{\mathrm{T}} \boldsymbol{\varphi}(s)+\boldsymbol{v}(s) .
$$

Referring to the decomposition methods in $[51,52,71]$, let $\boldsymbol{\psi}_{i}^{\mathrm{T}}(s) \in \mathbb{R}^{n_{0}}$ be the $i$ th row of the information matrix $\psi(s)$, that is

$$
\boldsymbol{\psi}(s):=\left[\boldsymbol{\psi}_{1}(s), \boldsymbol{\psi}_{2}(s), \cdots, \boldsymbol{\psi}_{m}(s)\right]^{\mathrm{T}} \in \mathbb{R}^{m \times n_{0}} .
$$

Similarly, let $\boldsymbol{\theta}_{i} \in \mathbb{R}^{r n}$ be the $i$ th column of the parameter matrix $\boldsymbol{\theta}$ :

$$
\boldsymbol{\theta}:=\left[\boldsymbol{\theta}_{1}, \boldsymbol{\theta}_{2}, \cdots, \boldsymbol{\theta}_{m}\right] \in \mathbb{R}^{(r n) \times m} .
$$

Then Equation (40) can be decomposed into $m$ subsystem identification models:

$$
\begin{aligned}
y_{i}(s) & =\boldsymbol{\psi}_{i}^{\mathrm{T}}(s) \boldsymbol{\beta}+\boldsymbol{\theta}_{i}^{\mathrm{T}} \boldsymbol{\varphi}(s)+v_{i}(s), \\
& =\boldsymbol{\psi}_{i}^{\mathrm{T}}(s) \boldsymbol{\beta}+\boldsymbol{\varphi}^{\mathrm{T}}(s) \boldsymbol{\theta}_{i}+v_{i}(s), \quad i=1,2, \cdots, m .
\end{aligned}
$$

According to the identification model in (41), define a gradient criterion function,

$$
J_{2}\left(\boldsymbol{\beta}, \boldsymbol{\theta}_{i}\right):=\sum_{j=1}^{s}\left[y_{i}(j)-\boldsymbol{\psi}_{i}^{\mathrm{T}}(j) \boldsymbol{\beta}-\boldsymbol{\varphi}^{\mathrm{T}}(j) \boldsymbol{\theta}_{i}\right]^{2}
$$

Let $\hat{\boldsymbol{\beta}}(s)$ and $\hat{\boldsymbol{\theta}}_{i}(s)$ be the estimates of $\boldsymbol{\beta}$ and $\boldsymbol{\theta}_{i}$ at time $s$. Minimizing $J_{2}\left(\boldsymbol{\beta}, \boldsymbol{\theta}_{i}\right)$ gives

$$
\begin{aligned}
& \left.\frac{\partial J_{2}\left(\boldsymbol{\beta}, \boldsymbol{\theta}_{i}\right)}{\partial \boldsymbol{\beta}}\right|_{\boldsymbol{\beta}=\hat{\boldsymbol{\beta}}(s)}=\left.\frac{\partial\left\|\boldsymbol{Y}_{i, s}-\boldsymbol{H}_{i, s} \boldsymbol{\beta}-\boldsymbol{Z}_{i, s} \boldsymbol{\theta}_{i}\right\|^{2}}{\partial \boldsymbol{\beta}}\right|_{\boldsymbol{\beta}=\hat{\boldsymbol{\beta}}(s)}=\mathbf{0} \\
& \left.\frac{\partial J_{2}\left(\boldsymbol{\beta}, \boldsymbol{\theta}_{i}\right)}{\partial \boldsymbol{\theta}_{i}}\right|_{\boldsymbol{\theta}_{i}=\hat{\boldsymbol{\theta}}_{i}(s)}=\left.\frac{\partial\left\|\boldsymbol{Y}_{i, s}-\boldsymbol{H}_{i, s} \boldsymbol{\beta}-\boldsymbol{Z}_{i, s} \boldsymbol{\theta}_{i}\right\|^{2}}{\partial \boldsymbol{\theta}_{i}}\right|_{\boldsymbol{\theta}_{i}=\hat{\boldsymbol{\theta}}_{i}(s)}=\mathbf{0}
\end{aligned}
$$

where

$$
\begin{aligned}
\boldsymbol{Y}_{i, s} & :=\left[y_{i}(1), y_{i}(2), y_{i}(3), \cdots, y_{i}(s)\right]^{\mathrm{T}} \in \mathbb{R}^{s}, \\
\boldsymbol{H}_{i, s} & :=\left[\boldsymbol{\psi}_{i}(1), \boldsymbol{\psi}_{i}(2), \boldsymbol{\psi}_{i}(3), \cdots, \boldsymbol{\psi}_{i}(s)\right]^{\mathrm{T}} \in \mathbb{R}^{s \times n_{0}}, \\
\boldsymbol{Z}_{i, s} & :=[\boldsymbol{\varphi}(1), \boldsymbol{\varphi}(2), \boldsymbol{\varphi}(3), \cdots, \boldsymbol{\varphi}(s)]^{\mathrm{T}} \in \mathbb{R}^{s \times(r n)},
\end{aligned}
$$

From (43) and (44), we can get the least squares estimates $\hat{\boldsymbol{\beta}}(s)$ and $\hat{\boldsymbol{\theta}}_{i}(s)$ of $\boldsymbol{\beta}$ and $\boldsymbol{\theta}_{i}$ :

$$
\begin{aligned}
\hat{\boldsymbol{\beta}}(s) & =\left(\boldsymbol{H}_{i, s}^{\mathrm{T}} \boldsymbol{H}_{i, s}\right)^{-1}\left(\boldsymbol{H}_{i, s}^{\mathrm{T}} \boldsymbol{Y}_{i, s}-\boldsymbol{H}_{i, s}^{\mathrm{T}} \boldsymbol{Z}_{i, s} \boldsymbol{\theta}_{i}\right), \\
& =\left[\sum_{j=1}^{s} \boldsymbol{\psi}_{i}(j) \boldsymbol{\psi}_{i}^{\mathrm{T}}(j)\right]^{-1}\left[\sum_{j=1}^{s} \boldsymbol{\psi}_{i}(j) y_{i}(j)-\sum_{j=1}^{s} \boldsymbol{\psi}_{i}(j) \boldsymbol{\varphi}^{\mathrm{T}}(j) \boldsymbol{\theta}_{i}\right],
\end{aligned}
$$




$$
\begin{aligned}
\hat{\boldsymbol{\theta}}_{i}(s) & =\left(\boldsymbol{Z}_{i, s}^{\mathrm{T}} \boldsymbol{Z}_{i, s}\right)^{-1}\left(\boldsymbol{Z}_{i, s}^{\mathrm{T}} \boldsymbol{Y}_{i, s}-\boldsymbol{Z}_{i, s}^{\mathrm{T}} \boldsymbol{H}_{i, s} \boldsymbol{\beta}\right), \\
& =\left[\sum_{j=1}^{s} \boldsymbol{\varphi}(j) \boldsymbol{\varphi}^{\mathrm{T}}(j)\right]^{-1}\left[\sum_{j=1}^{s} \boldsymbol{\varphi}(j) y_{i}(j)-\sum_{j=1}^{s} \boldsymbol{\varphi}(j) \boldsymbol{\psi}_{i}^{\mathrm{T}}(j) \boldsymbol{\beta}\right] .
\end{aligned}
$$

Define the covariance matrixes $\boldsymbol{P}_{\beta, i}(s)$ and $\boldsymbol{P}_{\theta, i}(s)$, and the gain matrixes $\boldsymbol{L}_{\beta, i}(s)$ and $\boldsymbol{L}_{\theta, i}(s)$ as

$$
\begin{aligned}
\boldsymbol{P}_{\beta, i}^{-1}(s) & :=\sum_{j=1}^{s} \boldsymbol{\psi}_{i}(j) \boldsymbol{\psi}_{i}^{\mathrm{T}}(j) \in \mathbb{R}^{n_{0} \times n_{0},} \\
& =\boldsymbol{P}_{\beta, i}^{-1}(s-1)+\boldsymbol{\psi}_{i}(s) \boldsymbol{\psi}_{i}^{\mathrm{T}}(s), \\
\boldsymbol{P}_{\theta, i}^{-1}(s) & :=\sum_{j=1}^{s} \boldsymbol{\varphi}(j) \boldsymbol{\varphi}^{\mathrm{T}}(j) \in \mathbb{R}^{(r n) \times(r n)}, \\
& =\boldsymbol{P}_{\theta, i}^{-1}(s-1)+\boldsymbol{\varphi}(s) \boldsymbol{\varphi}^{\mathrm{T}}(s), \\
\boldsymbol{L}_{\beta, i}(s) & :=\boldsymbol{P}_{\beta, i}(s) \boldsymbol{\psi}_{i}(s) \in \mathbb{R}^{n_{0}}, \\
\boldsymbol{L}_{\theta, i}(s) & :=\boldsymbol{P}_{\theta, i}(s) \boldsymbol{\varphi}(s) \in \mathbb{R}^{r n} .
\end{aligned}
$$

Based on the derivation of the RLS algorithm in [72,73], we can summarize the RLS estimates $\hat{\beta}(s)$ and $\hat{\boldsymbol{\theta}}_{i}(s)$ of $\boldsymbol{\beta}_{i}$ and $\boldsymbol{\theta}_{i}$ :

$$
\begin{aligned}
\hat{\boldsymbol{\beta}}(s) & =\hat{\boldsymbol{\beta}}(s-1)+\boldsymbol{L}_{\beta, i}(s)\left[y_{i}(s)-\boldsymbol{\psi}_{i}^{\mathrm{T}}(s) \hat{\boldsymbol{\beta}}(s-1)-\boldsymbol{\varphi}^{\mathrm{T}}(s) \hat{\boldsymbol{\theta}}_{i}(s-1)\right], \\
\boldsymbol{L}_{\beta, i}(s) & =\frac{\boldsymbol{P}_{\beta, i}(s-1) \boldsymbol{\psi}_{i}(s)}{1+\boldsymbol{\psi}_{i}^{\mathrm{T}}(s) \boldsymbol{P}_{\beta, i}(s-1) \boldsymbol{\psi}_{i}(s)} \\
\boldsymbol{P}_{\beta, i}(s) & =\boldsymbol{P}_{\beta, i}(s-1)-\boldsymbol{L}_{\beta, i}(s)\left[\boldsymbol{P}_{\beta, i}(s-1) \boldsymbol{\psi}_{i}(s)\right]^{\mathrm{T}}, \\
\hat{\boldsymbol{\theta}}_{i}(s) & =\hat{\boldsymbol{\theta}}_{i}(s-1)+\boldsymbol{L}_{\theta, i}(s)\left[y_{i}(s)-\boldsymbol{\psi}_{i}^{\mathrm{T}}(s) \hat{\boldsymbol{\beta}}(s-1)-\boldsymbol{\varphi}^{\mathrm{T}}(s) \hat{\boldsymbol{\theta}}_{i}(s-1)\right], \\
\boldsymbol{L}_{\theta, i}(s) & =\frac{\boldsymbol{P}_{\theta, i}(s-1) \boldsymbol{\varphi}(s)}{1+\boldsymbol{\varphi}^{\mathrm{T}}(s) \boldsymbol{P}_{\theta, i}(s-1) \boldsymbol{\varphi}(s)} \\
\boldsymbol{P}_{\theta, i}(s) & =\boldsymbol{P}_{\theta, i}(s-1)-\boldsymbol{L}_{\theta, i}(s)\left[\boldsymbol{P}_{\theta, i}(s-1) \boldsymbol{\varphi}(s)\right]^{\mathrm{T}} .
\end{aligned}
$$

However, Equations (55)-(60) cannot figure out the estimates $\hat{\boldsymbol{\beta}}(s)$ and $\hat{\boldsymbol{\theta}}_{i}(s)$. Because the subsystem information vector $\psi_{i}(s)$ includes the unknown vectors $x(s-i), \boldsymbol{w}(s-i)$ and $\boldsymbol{v}(s-i)$. In order to deal with this problem, we replace these unknown vectors in $\psi_{i}(s)$ with their corresponding estimates $\hat{x}(s-i), \hat{\boldsymbol{v}}(s-i)$ and $\hat{\boldsymbol{v}}(s-i)$ by making use of the auxiliary model identification idea. Then the estimates of $\psi(s), \psi_{s}(s)$ and $\psi_{n}(s)$ can be constructed by

$$
\begin{aligned}
\hat{\psi}(s) & :=\left[\hat{\boldsymbol{\psi}}_{s}(s), \hat{\boldsymbol{\psi}}_{n}(s)\right], \\
& =\left[\hat{\boldsymbol{\psi}}_{1}(s), \hat{\boldsymbol{\psi}}_{2}(s), \cdots, \hat{\boldsymbol{\psi}}_{m}(s)\right]^{\mathrm{T}} \in \mathbb{R}^{m \times n_{0}}, \\
\hat{\psi}_{s}(s) & :=[-\hat{\boldsymbol{x}}(s-1),-\hat{\boldsymbol{x}}(s-2), \cdots,-\hat{\boldsymbol{x}}(s-n)] \in \mathbb{R}^{m \times n} \\
\hat{\boldsymbol{\psi}}_{n}(s) & :=\left[-\hat{\boldsymbol{w}}(s-1), \cdots,-\hat{\boldsymbol{w}}\left(s-n_{c}\right), \hat{\boldsymbol{v}}(s-1), \cdots, \hat{\boldsymbol{v}}\left(s-n_{d}\right)\right] \in \mathbb{R}^{m \times n_{1}}
\end{aligned}
$$

Based on (4), (6) and (41), the estimates $\hat{x}(s), \hat{\boldsymbol{w}}(s)$ and $\hat{v}_{i}(s)$ at time $s$ can be calculated through three auxiliary models:

$$
\begin{aligned}
\hat{\boldsymbol{x}}(s) & :=\hat{\boldsymbol{\psi}}_{s}(s) \hat{\boldsymbol{\alpha}}(s)+\hat{\boldsymbol{\theta}}^{\mathrm{T}}(s) \boldsymbol{\varphi}(s) \\
\hat{\boldsymbol{w}}(s) & :=\boldsymbol{y}(s)-\hat{\boldsymbol{\psi}}_{s}(s) \hat{\boldsymbol{\alpha}}(s)-\hat{\boldsymbol{\theta}}^{\mathrm{T}}(s) \boldsymbol{\varphi}(s) \\
\hat{v}_{i}(s) & :=y_{i}(s)-\hat{\boldsymbol{\psi}}_{i}^{\mathrm{T}}(s) \hat{\boldsymbol{\beta}}(s)-\boldsymbol{\varphi}^{\mathrm{T}}(s) \hat{\boldsymbol{\theta}}_{i}(s)
\end{aligned}
$$


For clarity, let $\hat{\beta}_{i}(s)$ represent the estimate of $\beta$ in Subsystem $i$ at time $s$. Combining (61)-(67) and replacing $\psi_{i}(s)$ in (55)-(60) with its estimate $\hat{\psi}_{i}(s)$ and the same parameter vector $\hat{\boldsymbol{\beta}}(s)$ in (55)-(60) with $\hat{\beta}_{i}(s)$ give the subsystem recursive generalized extended least squares (S-RGELS) algorithm:

$$
\begin{aligned}
\hat{\boldsymbol{\beta}}_{i}(s) & =\hat{\boldsymbol{\beta}}_{i}(s-1)+\boldsymbol{L}_{\beta, i}(s)\left[y_{i}(s)-\hat{\boldsymbol{\psi}}_{i}^{\mathrm{T}}(s) \hat{\boldsymbol{\beta}}_{i}(s-1)-\boldsymbol{\varphi}^{\mathrm{T}}(s) \hat{\boldsymbol{\theta}}_{i}(s-1)\right], \quad i=1,2, \cdots, m, \\
\boldsymbol{L}_{\beta, i}(s) & =\boldsymbol{P}_{\beta, i}(s-1) \hat{\boldsymbol{\psi}}_{i}(s)\left[1+\hat{\boldsymbol{\psi}}_{i}^{\mathrm{T}}(s) \boldsymbol{P}_{\beta, i}(s-1) \hat{\boldsymbol{\psi}}_{i}(s)\right]^{-1}, \\
\boldsymbol{P}_{\beta, i}(s) & =\boldsymbol{P}_{\beta, i}(s-1)-\boldsymbol{L}_{\beta, i}(s)\left[\boldsymbol{P}_{\beta, i}(s-1) \hat{\boldsymbol{\psi}}_{i}(s)\right]^{\mathrm{T}}, \\
\hat{\boldsymbol{\theta}}_{i}(s) & =\hat{\boldsymbol{\theta}}_{i}(s-1)+\boldsymbol{L}_{\theta, i}(s)\left[y_{i}(s)-\hat{\boldsymbol{\psi}}_{i}^{\mathrm{T}}(s) \hat{\boldsymbol{\beta}}_{i}(s-1)-\boldsymbol{\varphi}^{\mathrm{T}}(s) \hat{\boldsymbol{\theta}}_{i}(s-1)\right], \\
\boldsymbol{L}_{\theta, i}(s) & =\boldsymbol{P}_{\theta, i}(s-1) \boldsymbol{\varphi}(s)\left[1+\boldsymbol{\varphi}^{\mathrm{T}}(s) \boldsymbol{P}_{\theta, i}(s-1) \boldsymbol{\varphi}(s)\right]^{-1}, \\
\boldsymbol{P}_{\theta, i}(s) & =\boldsymbol{P}_{\theta, i}(s-1)-\boldsymbol{L}_{\theta, i}(s)\left[\boldsymbol{P}_{\theta, i}(s-1) \boldsymbol{\varphi}(s)\right]^{\mathrm{T}}, \\
\boldsymbol{\varphi}(s) & =\left[\boldsymbol{u}^{\mathrm{T}}(s-1), \boldsymbol{u}^{\mathrm{T}}(s-2), \cdots, \boldsymbol{u}^{\mathrm{T}}(s-n)\right]^{\mathrm{T}}, \\
\hat{\boldsymbol{\psi}}(s) & =\left[\hat{\boldsymbol{\psi}}_{s}(s), \hat{\boldsymbol{\psi}}_{n}(s)\right] \\
& =\left[\hat{\boldsymbol{\psi}}_{1}(s), \hat{\boldsymbol{\psi}}_{2}(s), \cdots, \hat{\boldsymbol{\psi}}_{m}(s)\right]^{\mathrm{T}}, \\
\hat{\boldsymbol{\psi}}_{s}(s) & =[-\hat{\boldsymbol{x}}(s-1),-\hat{\boldsymbol{x}}(s-2), \cdots,-\hat{\boldsymbol{x}}(s-n)], \\
\hat{\boldsymbol{x}_{n}}(s) & =\left[-\hat{\boldsymbol{w}}(s-1),-\hat{\boldsymbol{w}}(s-2), \cdots,-\hat{\boldsymbol{w}}\left(s-n_{c}\right), \hat{\boldsymbol{v}}(s-1), \hat{\boldsymbol{v}}(s-2), \cdots, \hat{\boldsymbol{v}}\left(s-n_{d}\right)\right], \\
\hat{\boldsymbol{w}}(s) & =\boldsymbol{y}(s)-\hat{\boldsymbol{\psi}}_{s}(s) \hat{\boldsymbol{\alpha}}(s)+\hat{\boldsymbol{\theta}}^{\mathrm{T}}(s) \boldsymbol{\varphi}(s), \\
\hat{\boldsymbol{v}}_{i}(s) & =y_{i}(s)-\hat{\boldsymbol{\psi}}_{i}^{\mathrm{T}}(s) \hat{\boldsymbol{\beta}}_{i}(s)-\boldsymbol{\varphi}^{\mathrm{T}}(s) \boldsymbol{\varphi}(s), \\
\hat{\boldsymbol{v}}(s) & =\left[\hat{\boldsymbol{\theta}}_{1}(s), \hat{v}_{2}(s), \cdots, \hat{v}_{m}(s)\right]^{\mathrm{T}}, \\
\hat{\boldsymbol{\theta}}(s) & =\left[\hat{\boldsymbol{\theta}}_{1}(s), \hat{\boldsymbol{\theta}}_{2}(s), \cdots, \hat{\boldsymbol{\theta}}_{m}(s)\right]
\end{aligned}
$$

From the S-RGELS algorithm in (68)-(83), we can acquire $m$ estimates $\hat{\boldsymbol{\beta}}_{i}$ (s) of $\boldsymbol{\beta}$ from (68)-(83), it leads to a lot of redundant computation. However, we only need one parameter estimate of $\beta$. In order to cut down the redundant parameter estimates and improve the parameter estimation accuracy, the first way is to take their average value as the estimate of $\beta$ :

$$
\hat{\boldsymbol{\beta}}(s)=\frac{\hat{\boldsymbol{\beta}}_{1}(s)+\hat{\boldsymbol{\beta}}_{2}(s)+\cdots+\hat{\boldsymbol{\beta}}_{m}(s)}{m} \in \mathbb{R}^{n_{0}} .
$$

Replacing $\hat{\beta}_{i}(s-1)$ in (68)-(83) with $\hat{\boldsymbol{\beta}}(s-1)$ gives the partially-coupled subsystem recursive generalized extended least squares (PC-S-RGELS) algorithm:

$$
\begin{aligned}
\hat{\boldsymbol{\beta}}_{i}(s) & =\hat{\boldsymbol{\beta}}(s-1)+\boldsymbol{L}_{\beta, i}(s)\left[y_{i}(s)-\hat{\boldsymbol{\psi}}_{i}^{\mathrm{T}}(s) \hat{\boldsymbol{\beta}}(s-1)-\boldsymbol{\varphi}^{\mathrm{T}}(s) \hat{\boldsymbol{\theta}}_{i}(s-1)\right], \quad i=1,2, \cdots, m, \\
\boldsymbol{L}_{\beta, i}(s) & =\boldsymbol{P}_{\beta, i}(s-1) \hat{\boldsymbol{\psi}}_{i}(s)\left[1+\hat{\boldsymbol{\psi}}_{i}^{\mathrm{T}}(s) \boldsymbol{P}_{\beta, i}(s-1) \hat{\boldsymbol{\psi}}_{i}(s)\right]^{-1}, \\
\boldsymbol{P}_{\beta, i}(s) & =\boldsymbol{P}_{\beta, i}(s-1)-\boldsymbol{L}_{\beta, i}(s)\left[\boldsymbol{P}_{\beta, i}(s-1) \hat{\boldsymbol{\psi}}_{i}(s)\right]^{\mathrm{T}}, \\
\hat{\boldsymbol{\theta}}_{i}(s) & =\hat{\boldsymbol{\theta}}_{i}(s-1)+\boldsymbol{L}_{\theta, i}(s)\left[y_{i}(s)-\hat{\boldsymbol{\psi}}_{i}^{\mathrm{T}}(s) \hat{\boldsymbol{\beta}}(s-1)-\boldsymbol{\varphi}^{\mathrm{T}}(s) \hat{\boldsymbol{\theta}}_{i}(s-1)\right], \\
\boldsymbol{L}_{\theta, i}(s) & =\boldsymbol{P}_{\theta, i}(s-1) \boldsymbol{\varphi}(s)\left[1+\boldsymbol{\varphi}^{\mathrm{T}}(s) \boldsymbol{P}_{\theta, i}(s-1) \boldsymbol{\varphi}(s)\right]^{-1}, \\
\boldsymbol{P}_{\theta, i}(s) & =\boldsymbol{P}_{\theta, i}(s-1)-\boldsymbol{L}_{\theta, i}(s)\left[\boldsymbol{P}_{\theta, i}(s-1) \boldsymbol{\varphi}(s)\right]^{\mathrm{T}}, \\
\boldsymbol{\varphi}(s) & =\left[\boldsymbol{u}^{\mathrm{T}}(s-1), \boldsymbol{u}^{\mathrm{T}}(s-2), \cdots, \boldsymbol{u}^{\mathrm{T}}(s-n)\right]^{\mathrm{T}}, \\
\hat{\boldsymbol{\psi}}(s) & =\left[\hat{\boldsymbol{\psi}}_{s}(s), \hat{\boldsymbol{\psi}}_{n}(s)\right] \\
& =\left[\hat{\boldsymbol{\psi}}_{1}(s), \hat{\boldsymbol{\psi}}_{2}(s), \cdots, \hat{\boldsymbol{\psi}}_{m}(s)\right]^{\mathrm{T}}, \\
\hat{\boldsymbol{\psi}}_{s}(s) & =[-\hat{\boldsymbol{x}}(s-1),-\hat{\boldsymbol{x}}(s-2), \cdots,-\hat{\boldsymbol{x}}(s-n)], \\
\hat{\boldsymbol{\psi}}_{n}(s) & =\left[-\hat{\boldsymbol{v}}(s-1),-\hat{\boldsymbol{w}}(s-2), \cdots,-\hat{\boldsymbol{v}}\left(s-n_{c}\right), \hat{\boldsymbol{v}}(s-1), \hat{\boldsymbol{v}}(s-2), \cdots, \hat{\boldsymbol{v}}\left(s-n_{d}\right)\right], \\
\hat{\boldsymbol{w}}(s) & =\boldsymbol{y}(s)-\hat{\boldsymbol{\psi}}_{s}(s) \hat{\boldsymbol{\alpha}}(s)+\hat{\boldsymbol{\theta}}^{\mathrm{T}}(s) \boldsymbol{\varphi}(s), \hat{\boldsymbol{\theta}}^{\mathrm{T}}(s) \boldsymbol{\varphi}(s)
\end{aligned}
$$




$$
\begin{aligned}
& \hat{\boldsymbol{v}}(s)=\boldsymbol{y}(s)-\hat{\boldsymbol{\psi}}(s) \hat{\boldsymbol{\beta}}(s)-\hat{\boldsymbol{\theta}}^{\mathrm{T}}(s) \boldsymbol{\varphi}(s), \\
& \hat{\boldsymbol{\beta}}(s)=\frac{\hat{\boldsymbol{\beta}}_{1}(s)+\hat{\boldsymbol{\beta}}_{2}(s)+\cdots+\hat{\boldsymbol{\beta}}_{m}(s)}{m} \\
& \hat{\boldsymbol{\theta}}(s)=\left[\hat{\boldsymbol{\theta}}_{1}(s), \hat{\boldsymbol{\theta}}_{2}(s), \cdots, \hat{\boldsymbol{\theta}}_{m}(s)\right] .
\end{aligned}
$$

Generally, for the recursive algorithms, it is considered that the parameter estimates are close to their true parameters as the time $s$ increasing. Therefore, the estimate $\hat{\beta}_{i-1}(s)$ is closer to the true parameter than the estimate $\hat{\boldsymbol{\beta}}_{i}(s-1)$. Combining (91)-(100) and replacing $\hat{\beta}_{i}(s-1)$ in (68) and (71) when $i=1$ with $\hat{\boldsymbol{\beta}}_{m}(s-1)$ and $\hat{\boldsymbol{\beta}}_{i}(s-1)$ in (68) and (71) when $i=2,3, \cdots, m$ with $\hat{\boldsymbol{\beta}}_{i-1}(s)$ give the partially-coupled recursive generalized extended least squares (PC-RGELS) algorithm:

$$
\begin{aligned}
& \hat{\boldsymbol{\beta}}_{1}(s)=\hat{\boldsymbol{\beta}}_{m}(s-1)+\boldsymbol{L}_{\beta, 1}(s)\left[y_{1}(s)-\hat{\boldsymbol{\psi}}_{1}^{\mathrm{T}}(s) \hat{\boldsymbol{\beta}}_{m}(s-1)-\boldsymbol{\varphi}^{\mathrm{T}}(s) \hat{\boldsymbol{\theta}}_{1}(s-1)\right], \\
& \boldsymbol{L}_{\beta, 1}(s)=\boldsymbol{P}_{\beta, 1}(s-1) \hat{\boldsymbol{\psi}}_{i}(s)\left[1+\hat{\boldsymbol{\psi}}_{1}^{\mathrm{T}}(s) \boldsymbol{P}_{\beta, 1}(s-1) \hat{\boldsymbol{\psi}}_{1}(s)\right]^{-1}, \\
& \boldsymbol{P}_{\beta, 1}(s)=\boldsymbol{P}_{\beta, 1}(s-1)-\boldsymbol{L}_{\beta, 1}(s)\left[\boldsymbol{P}_{\beta, 1}(s-1) \hat{\psi}_{1}(s)\right]^{\mathrm{T}}, \\
& \hat{\boldsymbol{\theta}}_{1}(s)=\hat{\boldsymbol{\theta}}_{1}(s-1)+\boldsymbol{L}_{\theta, 1}(s)\left[y_{1}(s)-\hat{\boldsymbol{\psi}}_{1}^{\mathrm{T}}(s) \hat{\boldsymbol{\beta}}_{m}(s-1)-\boldsymbol{\varphi}^{\mathrm{T}}(s) \hat{\boldsymbol{\theta}}_{1}(s-1)\right], \\
& \boldsymbol{L}_{\theta, 1}(s)=\boldsymbol{P}_{\theta, 1}(s-1) \boldsymbol{\varphi}(s)\left[1+\boldsymbol{\varphi}^{\mathrm{T}}(s) \boldsymbol{P}_{\theta, 1}(s-1) \boldsymbol{\varphi}(s)\right]^{-1}, \\
& \boldsymbol{P}_{\theta, 1}(s)=\boldsymbol{P}_{\theta, 1}(s-1)-\boldsymbol{L}_{\theta, i}(s)\left[\boldsymbol{P}_{\theta, 1}(s-1) \boldsymbol{\varphi}(s)\right]^{\mathrm{T}} \text {, } \\
& \hat{\boldsymbol{\beta}}_{i}(s)=\hat{\boldsymbol{\beta}}_{i-1}(s)+\boldsymbol{L}_{\beta, i}(s)\left[y_{i}(s)-\hat{\boldsymbol{\psi}}_{i}^{\mathrm{T}}(s) \hat{\boldsymbol{\beta}}_{i-1}(s)-\boldsymbol{\varphi}^{\mathrm{T}}(s) \hat{\boldsymbol{\theta}}_{i}(s-1)\right], \quad i=2,3, \cdots, m, \\
& \boldsymbol{L}_{\beta, i}(s)=\boldsymbol{P}_{\beta, i}(s-1) \hat{\boldsymbol{\psi}}_{i}(s)\left[1+\hat{\boldsymbol{\psi}}_{i}^{\mathrm{T}}(s) \boldsymbol{P}_{\beta, i}(s-1) \hat{\boldsymbol{\psi}}_{i}(s)\right]^{-1}, \\
& \boldsymbol{P}_{\beta, i}(s)=\boldsymbol{P}_{\beta, i}(s-1)-\boldsymbol{L}_{\beta, i}(s)\left[\boldsymbol{P}_{\beta, i}(s-1) \hat{\boldsymbol{\psi}}_{i}(s)\right]^{\mathrm{T}} \text {, } \\
& \hat{\boldsymbol{\theta}}_{i}(s)=\hat{\boldsymbol{\theta}}_{i}(s-1)+\boldsymbol{L}_{\theta, i}(s)\left[y_{i}(s)-\hat{\boldsymbol{\psi}}_{i}^{\mathrm{T}}(s) \hat{\boldsymbol{\beta}}_{i-1}(s)-\boldsymbol{\varphi}^{\mathrm{T}}(s) \hat{\boldsymbol{\theta}}_{i}(s-1)\right], \\
& \boldsymbol{L}_{\theta, i}(s)=\boldsymbol{P}_{\theta, i}(s-1) \boldsymbol{\varphi}(s)\left[1+\boldsymbol{\varphi}^{\mathrm{T}}(s) \boldsymbol{P}_{\theta, i}(s-1) \boldsymbol{\varphi}(s)\right]^{-1} \text {, } \\
& \boldsymbol{P}_{\theta, i}(s)=\boldsymbol{P}_{\theta, i}(s-1)-\boldsymbol{L}_{\theta, i}(s)\left[\boldsymbol{P}_{\theta, i}(s-1) \boldsymbol{\varphi}(s)\right]^{\mathrm{T}} \text {, } \\
& \boldsymbol{\varphi}(s)=\left[\boldsymbol{u}^{\mathrm{T}}(s-1), \boldsymbol{u}^{\mathrm{T}}(s-2), \cdots, \boldsymbol{u}^{\mathrm{T}}(s-n)\right]^{\mathrm{T}}, \\
& \hat{\psi}(s)=\left[\hat{\psi}_{s}(s), \hat{\psi}_{n}(s)\right] \text {, } \\
& =\left[\hat{\psi}_{1}(s), \hat{\psi}_{2}(s), \cdots, \hat{\psi}_{m}(s)\right]^{\mathrm{T}} \text {, } \\
& \hat{\psi}_{s}(s)=[-\hat{x}(s-1),-\hat{x}(s-2), \cdots,-\hat{x}(s-n)], \\
& \hat{\psi}_{n}(s)=\left[-\hat{\boldsymbol{w}}(s-1),-\hat{\boldsymbol{w}}(s-2), \cdots,-\hat{\boldsymbol{w}}\left(s-n_{c}\right), \hat{\boldsymbol{v}}(s-1), \hat{\boldsymbol{v}}(s-2), \cdots, \hat{\boldsymbol{v}}\left(s-n_{d}\right)\right], \\
& \hat{\boldsymbol{x}}(s)=\hat{\boldsymbol{\psi}}_{s}(s) \hat{\boldsymbol{\alpha}}(s)+\hat{\boldsymbol{\theta}}^{\mathrm{T}}(s) \boldsymbol{\varphi}(s), \\
& \hat{\boldsymbol{w}}(s)=\boldsymbol{y}(s)-\hat{\boldsymbol{\psi}}_{s}(s) \hat{\boldsymbol{\alpha}}(s)-\hat{\boldsymbol{\theta}}^{\mathrm{T}}(s) \boldsymbol{\varphi}(s), \\
& \hat{v}_{i}(s)=y_{i}(s)-\hat{\boldsymbol{\psi}}_{i}^{\mathrm{T}}(s) \hat{\boldsymbol{\beta}}_{m}(s)-\boldsymbol{\varphi}^{\mathrm{T}}(s) \hat{\boldsymbol{\theta}}_{i}(s), \\
& \hat{v}(s)=\left[\hat{v}_{1}(s), \hat{v}_{2}(s), \cdots, \hat{v}_{m}(s)\right]^{\mathrm{T}} \text {, } \\
& \hat{\boldsymbol{\theta}}(s)=\left[\hat{\boldsymbol{\theta}}_{1}(s), \hat{\boldsymbol{\theta}}_{2}(s), \cdots, \hat{\boldsymbol{\theta}}_{m}(s)\right] \text {. }
\end{aligned}
$$

The procedures for achieving the PC-RGELS algorithm in (101)-(122) are as follows.

1. For $s \leqslant 0$, all variables are set to zero. Set the data length $L$. Let $s=1$, set the initial values $\hat{\boldsymbol{\beta}}_{m}(0)=\mathbf{1}_{n_{0}} / p_{0}, \hat{\boldsymbol{\theta}}_{i}(0)=\mathbf{1}_{r n} / p_{0}, \hat{\boldsymbol{x}}(0)=\mathbf{1}_{m} / p_{0}, \hat{\boldsymbol{w}}(0)=\mathbf{1}_{m} / p_{0}, \hat{\boldsymbol{v}}(0)=\mathbf{1}_{m} / p_{0}, \boldsymbol{P}_{\beta, i}(0)=p_{0} \boldsymbol{I}_{n_{0}}$, $\boldsymbol{P}_{\theta, i}(0)=p_{0} \boldsymbol{I}_{r n}, p_{0}=10^{6}$

2. Collect the input-output data $\boldsymbol{u}(s)=\left[u_{1}(s), u_{2}(s), \cdots, u_{r}(s)\right]^{\mathrm{T}}$ and $\boldsymbol{y}(s)=\left[y_{1}(s), y_{2}(s), \cdots\right.$, $\left.y_{m}(s)\right]^{\mathrm{T}}$, and construct $\boldsymbol{\varphi}(s)$ using (113).

3. Form $\hat{\psi}_{s}(s)$ and $\hat{\psi}_{n}(s)$ using (116)-(117) and construct $\hat{\psi}(s)$ using (114), and read $\hat{\psi}_{i}(s)$ from $\hat{\psi}(s)$ in (115), $i=1,2,3, \cdots, m$.

4. Compute $\boldsymbol{P}_{\beta, 1}(s)$ and $\boldsymbol{P}_{\theta, 1}(s)$ using (103) and (106), and compute $\boldsymbol{L}_{\beta, 1}(s)$ and $\boldsymbol{L}_{\theta, 1}(s)$ using (102) and (105), and update the estimates $\hat{\boldsymbol{\beta}}_{1}(s)$ and $\hat{\boldsymbol{\theta}}_{1}(s)$ using (101) and (104). 
5. For $i=2,3, \cdots, m$, calculate $\boldsymbol{P}_{\beta, i}(s)$ and $\boldsymbol{P}_{\theta, i}(s)$ using (109) and (112), and compute $\boldsymbol{L}_{\boldsymbol{\beta}, i}(s)$ and $\boldsymbol{L}_{\theta, i}(s)$ using (108) and (111), and refresh the estimates $\hat{\boldsymbol{\beta}}_{i}(s)$ and $\hat{\boldsymbol{\theta}}_{i}(s)$ using (107) and (110).

6. Construct $\hat{\boldsymbol{\theta}}(s)$ by (122), calculate the estimates $\hat{\boldsymbol{x}}(s), \hat{\boldsymbol{w}}(s)$ and $\hat{\boldsymbol{v}}(s)$ using (118)-(120).

7. Compare $s$ with $L$ : if $s \leqslant L$, increase $s$ by 1 and go to Step 2; otherwise obtain the estimate $\hat{\boldsymbol{\beta}}(L)$ and $\hat{\boldsymbol{\theta}}(L)$ and terminate this procedure.

The flowchart of computing $\hat{\boldsymbol{\beta}}(L)$ and $\hat{\boldsymbol{\theta}}(L)$ in the PC-RGELS algorithm is shown in Figure 2.

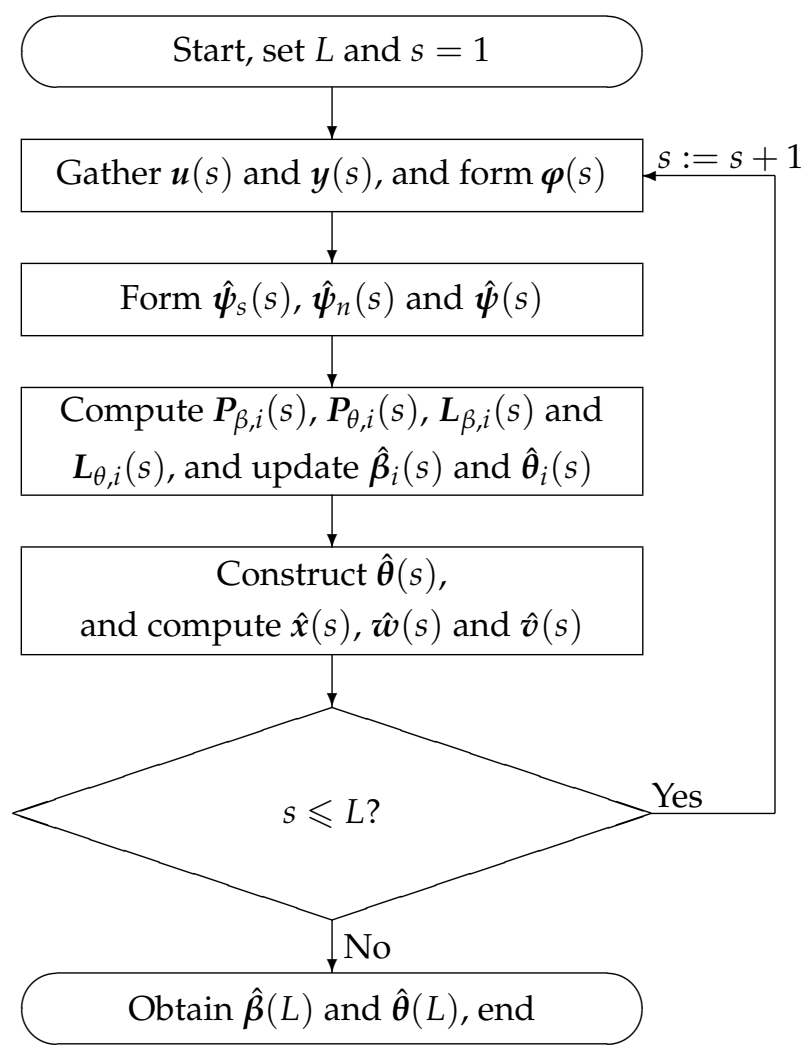

Figure 2. The flowchart of computing the PC-RGELS parameter estimates $\hat{\boldsymbol{\beta}}(L)$ and $\hat{\boldsymbol{\theta}}(L)$.

\section{Example}

The numerical simulation is based on the following M-OEARMA-like system:

$$
\begin{aligned}
y(s) & =\frac{Q(z)}{\alpha(z)} \boldsymbol{u}(s)+\frac{D(z)}{C(z)} \boldsymbol{v}(s), \\
\alpha(z) & :=1+a_{1} z^{-1}=1+0.26 z^{-1}, \\
Q(z) & :=A_{1} z^{-1}=\left[\begin{array}{ll}
a_{2} & a_{3} \\
a_{4} & a_{5}
\end{array}\right] z^{-1}=\left[\begin{array}{cc}
-0.57 z^{-1} & 0.93 z^{-1} \\
0.87 z^{-1} & 0.65^{-1}
\end{array}\right], \\
C(z) & :=1+c_{1} z^{-1}=1-0.56 z^{-1}, \\
D(z) & :=1+d_{1} z^{-1}=1+0.32 z^{-1},
\end{aligned}
$$

the parameter vectors of the system are

$$
\begin{aligned}
\boldsymbol{\alpha} & :=\left[a_{1}, a_{2}, a_{3}, a_{4}, a_{5}\right]^{\mathrm{T}}=[0.26,-0.57,0.93,0.87,0.65]^{\mathrm{T}}, \\
\boldsymbol{\beta} & :=\left[c_{1}, d_{1}\right]^{\mathrm{T}}=[-0.56,0.32]^{\mathrm{T}}, \\
\boldsymbol{\vartheta} & :=\left[\begin{array}{c}
\boldsymbol{\alpha} \\
\boldsymbol{\beta}
\end{array}\right]=\left[a_{1}, a_{2}, a_{3}, a_{4}, a_{5}, c_{1}, d_{1}\right]^{\mathrm{T}}, \\
& =[0.26,-0.57,0.93,0.87,0.65,-0.56,0.32]^{\mathrm{T}} .
\end{aligned}
$$


Here, the inputs $\left\{u_{1}(s)\right\}$ and $\left\{u_{2}(s)\right\}$ are taken as two independent persistent excitation signal sequences with zero mean and unit variances, $\left\{v_{1}(s)\right\}$ and $\left\{v_{2}(s)\right\}$ are taken as two white noise sequences with zero mean and variances $\sigma_{1}^{2}$ for $v_{1}(s)$ and $\sigma_{2}^{2}$ for $v_{2}(s)$. Set $\sigma_{1}^{2}=\sigma_{2}^{2}=0.30^{2}$. Then we can use them to generate the output vector $y(s)=\left[y_{1}(s), y_{2}(s)\right]^{\mathrm{T}}$. Set the data length $L=3000$. Applying the RGELS, PC-S-RGELS and PC-RGELS algorithms to identy the parameters of the given system. The parameter estimates and their estimation errors are shown in Tables 1-3. The parameter estimation errors $\delta:=\|\hat{\boldsymbol{\theta}}(s)-\boldsymbol{\theta}\| /\|\boldsymbol{\theta}\|$ versus $s$ is shown in Figure 3 .

Table 1. The RGELS estimates and their errors.

\begin{tabular}{ccccccccc}
\hline $\boldsymbol{s}$ & $\boldsymbol{a}_{\mathbf{1}}$ & $\boldsymbol{a}_{\mathbf{2}}$ & $\boldsymbol{a}_{\mathbf{3}}$ & $\boldsymbol{a}_{\mathbf{4}}$ & $\boldsymbol{a}_{\mathbf{5}}$ & $\boldsymbol{c}_{\mathbf{1}}$ & $\boldsymbol{d}_{\mathbf{1}}$ & $\boldsymbol{\delta}(\boldsymbol{\%})$ \\
\hline 100 & 0.21713 & -0.55471 & 0.90627 & 0.84332 & 0.71040 & -0.48095 & 0.18013 & 10.72413 \\
200 & 0.27110 & -0.56221 & 0.94490 & 0.86938 & 0.64018 & -0.47391 & 0.23325 & 7.35692 \\
500 & 0.24836 & -0.54410 & 0.93994 & 0.86873 & 0.66404 & -0.55063 & 0.06758 & 15.08382 \\
1000 & 0.26144 & -0.55626 & 0.94061 & 0.86873 & 0.64676 & -0.59024 & 0.03508 & 16.99606 \\
2000 & 0.25755 & -0.54862 & 0.94853 & 0.86843 & 0.65790 & -0.57538 & 0.03620 & 16.91802 \\
3000 & 0.25925 & -0.55582 & 0.94269 & 0.86686 & 0.66788 & -0.56765 & 0.05916 & 15.52793 \\
\hline True values & 0.26000 & -0.57000 & 0.93000 & 0.87000 & 0.65000 & -0.56000 & 0.32000 & \\
\hline
\end{tabular}

Table 2. The PC-S-RGELS estimates and their errors.

\begin{tabular}{ccccccccc}
\hline $\boldsymbol{s}$ & $\boldsymbol{a}_{\mathbf{1}}$ & $\boldsymbol{a}_{\mathbf{2}}$ & $\boldsymbol{a}_{\mathbf{3}}$ & $\boldsymbol{a}_{\mathbf{4}}$ & $\boldsymbol{a}_{\mathbf{5}}$ & $\boldsymbol{c}_{\mathbf{1}}$ & $\boldsymbol{d}_{\mathbf{1}}$ & $\boldsymbol{\delta}(\boldsymbol{\%})$ \\
\hline 100 & 0.20140 & -0.56387 & 0.92631 & 0.70446 & 0.79768 & -0.35762 & 0.68920 & 28.39191 \\
200 & 0.24432 & -0.56531 & 0.93373 & 0.79687 & 0.69082 & -0.38330 & 0.64577 & 22.51734 \\
500 & 0.25446 & -0.56183 & 0.94070 & 0.83956 & 0.67391 & -0.47448 & 0.47151 & 10.58749 \\
1000 & 0.26117 & -0.56373 & 0.93438 & 0.85347 & 0.65752 & -0.53154 & 0.40254 & 5.29978 \\
2000 & 0.25977 & -0.55982 & 0.93912 & 0.86161 & 0.65870 & -0.54046 & 0.37127 & 3.42342 \\
3000 & 0.26036 & -0.56350 & 0.93576 & 0.86339 & 0.66214 & -0.54830 & 0.37027 & 3.20492 \\
\hline True values & 0.26000 & -0.57000 & 0.93000 & 0.87000 & 0.65000 & -0.56000 & 0.32000 & \\
\hline
\end{tabular}

Table 3. The PC-RGELS estimates and their errors.

\begin{tabular}{ccccccccc}
\hline $\boldsymbol{s}$ & $\boldsymbol{a}_{\mathbf{1}}$ & $\boldsymbol{a}_{\mathbf{2}}$ & $\boldsymbol{a}_{\mathbf{3}}$ & $\boldsymbol{a}_{\mathbf{4}}$ & $\boldsymbol{a}_{\mathbf{5}}$ & $\boldsymbol{c}_{\mathbf{1}}$ & $\boldsymbol{d}_{\mathbf{1}}$ & $\boldsymbol{\delta}(\boldsymbol{\%})$ \\
\hline 100 & 0.24978 & -0.60667 & 0.87031 & 0.78115 & 0.56724 & -0.39507 & 0.30167 & 12.87537 \\
200 & 0.27882 & -0.58559 & 0.91259 & 0.82014 & 0.61386 & -0.42910 & 0.41254 & 10.32160 \\
500 & 0.25835 & -0.56532 & 0.93012 & 0.85414 & 0.64496 & -0.58010 & 0.25299 & 4.26796 \\
1000 & 0.26982 & -0.56619 & 0.93045 & 0.85993 & 0.64143 & -0.60694 & 0.26914 & 4.21835 \\
2000 & 0.26104 & -0.56177 & 0.93780 & 0.86480 & 0.65036 & -0.57656 & 0.30440 & 1.53744 \\
3000 & 0.26119 & -0.56487 & 0.93488 & 0.86510 & 0.65647 & -0.56786 & 0.34146 & 1.49741 \\
\hline True values & 0.26000 & -0.57000 & 0.93000 & 0.87000 & 0.65000 & -0.56000 & 0.32000 & \\
\hline
\end{tabular}

Taking the simulation conditions with $\sigma_{1}^{2}=\sigma_{2}^{2}=0.20^{2}$ and $\sigma_{1}^{2}=\sigma_{2}^{2}=0.60^{2}$, using the PC-RGELS algorithm to identify the parameters of the given systems, respectively, the parameter estimates and their estimation errors are shown in Tables 4 and 5 , and the estimation errors $\delta$ versus $s$ is shown in Figure 4 .

Table 4. The PC-RGELS estimates and their errors with $\sigma^{2}=0.20^{2}$.

\begin{tabular}{ccccccccc}
\hline $\boldsymbol{s}$ & $\boldsymbol{a}_{\mathbf{1}}$ & $\boldsymbol{a}_{\mathbf{2}}$ & $\boldsymbol{a}_{\mathbf{3}}$ & $\boldsymbol{a}_{\mathbf{4}}$ & $\boldsymbol{a}_{\mathbf{5}}$ & $\boldsymbol{c}_{\mathbf{1}}$ & $\boldsymbol{d}_{\mathbf{1}}$ & $\boldsymbol{\delta}(\boldsymbol{\%})$ \\
\hline 100 & 0.24988 & -0.56806 & 0.90461 & 0.89323 & 0.60879 & -0.59192 & 0.23442 & 6.30236 \\
200 & 0.26570 & -0.55866 & 0.90290 & 0.89140 & 0.62628 & -0.56227 & 0.30809 & 2.68948 \\
500 & 0.26807 & -0.57330 & 0.92228 & 0.86927 & 0.62782 & -0.55656 & 0.31763 & 1.50427 \\
1000 & 0.26418 & -0.57144 & 0.92234 & 0.87348 & 0.63193 & -0.56993 & 0.32145 & 1.34724 \\
2000 & 0.26205 & -0.57095 & 0.92456 & 0.87546 & 0.64561 & -0.54789 & 0.32853 & 1.03059 \\
3000 & 0.26050 & -0.56927 & 0.92491 & 0.87164 & 0.64979 & -0.54752 & 0.31805 & 0.81408 \\
\hline True values & 0.26000 & -0.57000 & 0.93000 & 0.87000 & 0.65000 & -0.56000 & 0.32000 & \\
\hline
\end{tabular}


Table 5. The PC-RGELS estimates and their errors with $\sigma^{2}=0.60^{2}$.

\begin{tabular}{ccccccccc}
\hline $\boldsymbol{s}$ & $\boldsymbol{a}_{\mathbf{1}}$ & $\boldsymbol{a}_{\mathbf{2}}$ & $\boldsymbol{a}_{\mathbf{3}}$ & $\boldsymbol{a}_{\mathbf{4}}$ & $\boldsymbol{a}_{\mathbf{5}}$ & $\boldsymbol{c}_{\mathbf{1}}$ & $\boldsymbol{d}_{\mathbf{1}}$ & $\boldsymbol{\delta}(\boldsymbol{\%})$ \\
\hline 100 & -0.07666 & -1.10323 & 1.30348 & 0.68217 & 0.72605 & -0.49016 & -0.09463 & 51.44481 \\
200 & 0.02363 & -0.82959 & 1.02696 & 0.84406 & 0.70880 & -0.49353 & -0.03312 & 30.52969 \\
500 & 0.16820 & -0.71220 & 1.00389 & 0.83283 & 0.64901 & -0.50435 & 0.06928 & 18.85790 \\
1000 & 0.20791 & -0.63623 & 0.95860 & 0.86602 & 0.63011 & -0.54443 & 0.15480 & 11.21196 \\
2000 & 0.23053 & -0.60174 & 0.94024 & 0.87889 & 0.65490 & -0.53307 & 0.25093 & 5.15448 \\
3000 & 0.24053 & -0.58686 & 0.93174 & 0.87014 & 0.66124 & -0.53546 & 0.27576 & 3.42763 \\
\hline True values & 0.26000 & -0.57000 & 0.93000 & 0.87000 & 0.65000 & -0.56000 & 0.32000 & \\
\hline
\end{tabular}

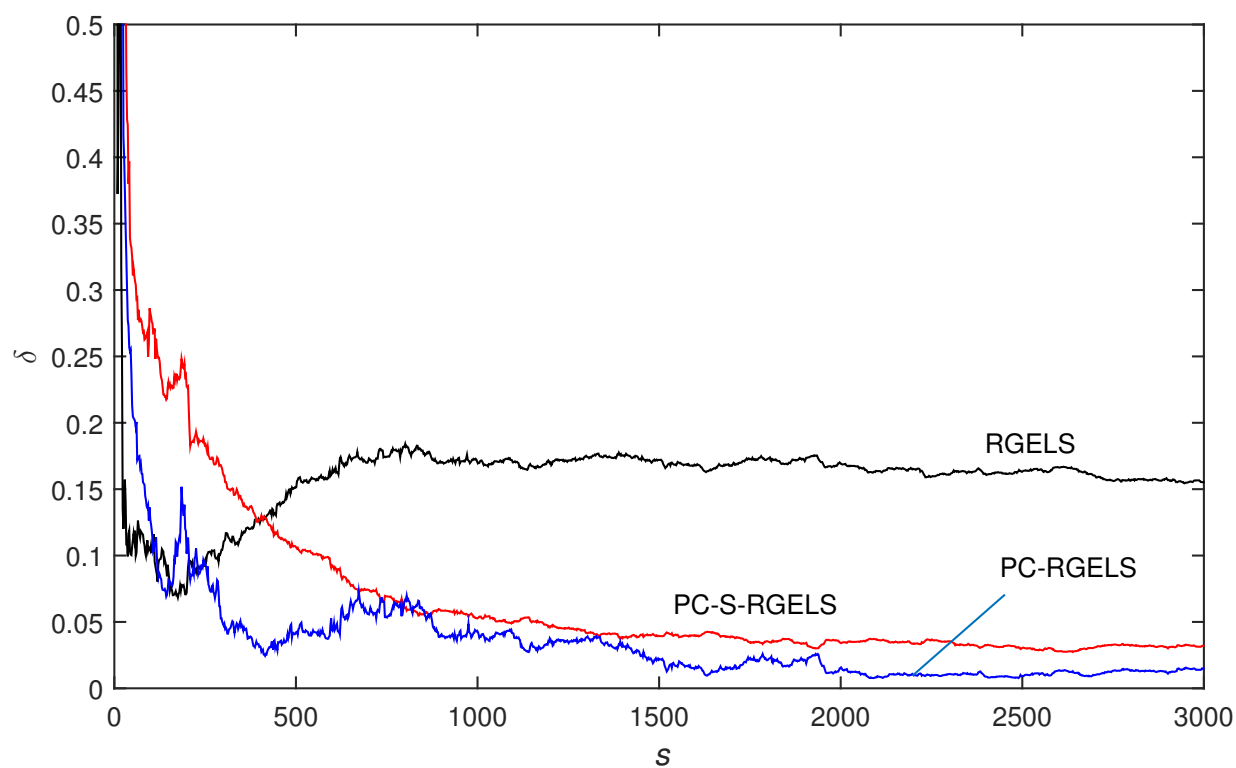

Figure 3. The RGELS, PC-S-RGELS and PC-RGELS estimation errors $\delta$ versus $s$.

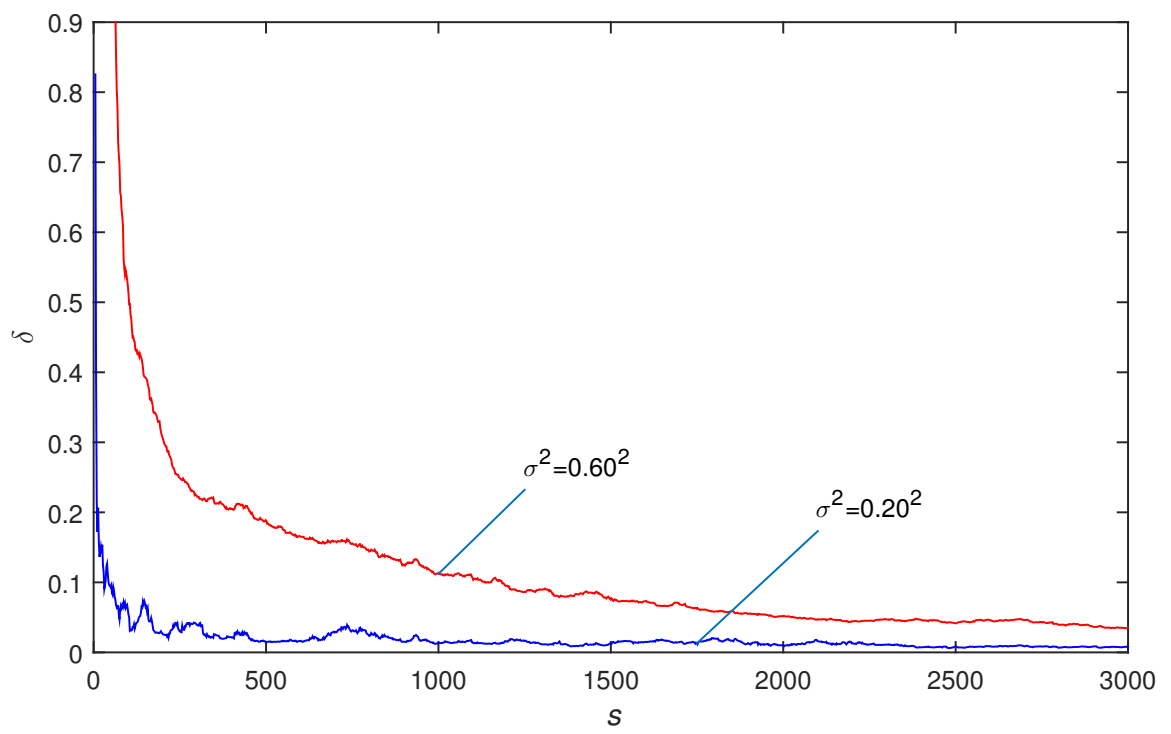

Figure 4. The PC-RGELS estimation errors $\delta$ versus $s$ with different $\sigma^{2}$.

From Tables 1-5 and Figures 3 and 4, we can draw the following conclusions.

- $\quad$ The parameter estimation errors given by the RGELS, PC-S-RGELS and PC-RGELS algorithms become smaller as $s$ increasing. Thus the proposed algorithms for multivariable OEARMA-like system are effective. 
- Under the same simulation conditions, the PC-S-RGELS and PC-RGELS algorithms can give more accurate parameter estimates compared with the RGELS algorithm.

- A lower noise level leads to a higher parameter estimation accuracy by the PC-RGELS algorithm under the same data length.

\section{Conclusions}

In this paper, we have dealt with the parameter identification problems of the M-OEARMA-like systems. A partially-coupled recursive generalized extended least squares algorithm is presented based on the hierarchical identification principle and the coupling identification concept. At the last of this paper, we discussed the comparison between the RGELS algorithm and the derived algorithms, and found that the PC-S-RGELS and PC-RGELS algorithms have higher computational efficiency than the RGELS algorithm on account of the proposed algorithms avoid calculating the inverse of the covariance matrix. The analysis and numerical example indicate that the PC-RGELS algorithm can give more accurate parameter estimates than the RGELS algorithm. Furthermore, the proposed methods can be extended to other fields by means of some other mathematical tools and approaches [80-86] to model industrial processes [87-98].

Author Contributions: Conceptualization and methodology, J.P., H.M. and F.D.; software, H.M. and L.L.; validation and analysis, G.X., A.A. and T.H. Finally, all the authors have read and approved the final manuscript.

Funding: This work was supported by the National Natural Science Foundation of China (no. 61603127) and the 111 Project (B12018).

Conflicts of Interest: The authors declare no conflict of interest.

\section{References}

1. Na, J.; Chen, A.S.; Herrmann, G.; Burke, R.; Brace, C. Vehicle engine torque estimation via unknown input observer and adaptive parameter estimation. IEEE Trans. Veh. Technol. 2018, 67, 409-422. [CrossRef]

2. Pan, J.; Li, W.; Zhang, H.P. Control algorithms of magnetic suspension systems based on the improved double exponential reaching law of sliding mode control. Int. J. Control Autom. Syst. 2018, 16, 2878-2887. [CrossRef]

3. Zhang, X.; Ding, F.; Xu, L.; Yang, E.F. Highly computationally efficient state filter based on the delta operator. Int. J. Adapt. Control Signal Process. 2019, 33, 875-889. [CrossRef]

4. $\mathrm{Xu}, \mathrm{H}$.; Ding, F.; Yang, E.F. Modeling a nonlinear process using the exponential autoregressive time series model. Nonlinear Dyn. 2019, 95, 2079-2092. [CrossRef]

5. Xu, L.; Chen, L.; Xiong, W.L. Parameter estimation and controller design for dynamic systems from the step responses based on the Newton iteration. Nonlinear Dyn. 2015, 79, 2155-2163. [CrossRef]

6. $\mathrm{Xu}, \mathrm{L}$. The parameter estimation algorithms based on the dynamical response measurement data. Adv. Mech. Eng. 2017, 9. [CrossRef]

7. Xu, L.; Ding, F.; Gu, Y.; Alsaedi, A.; Hayat, T. A multi-innovation state and parameter estimation algorithm for a state space system with d-step state-delay. Signal Process. 2017, 140, 97-103. [CrossRef]

8. $\mathrm{Xu}, \mathrm{L}$; Ding, F. Iterative parameter estimation for signal models based on measured data. Circuits Syst. Signal Process. 2018, 37, 3046-3069. [CrossRef]

9. Zhang, W.; Lin, X.; Chen, B.S. LaSalle-type theorem and its applications to infinite horizon optimal control of discrete-time nonlinear stochastic systems. IEEE Trans. Autom. Control 2017, 62, 250-261. [CrossRef]

10. Li, N.; Guo, S.; Wang, Y. Weighted preliminary-summation-based principal component analysis for non-Gaussian processes. Control Eng. Pract. 2019, 87, 122-132. [CrossRef]

11. Wang, Y.; Si, Y.; Huang, B.; Lou, Z. Survey on the theoretical research and engineering applications of multivariate statistics process monitoring algorithms: 2008-2017. Can. J. Chem. Eng. 2018, 96, 2073-2085. [CrossRef]

12. Wang, Y.Q.; Zhang, H.; Wei, S.L.; Zhou, D.G.; Huang, B. Control performance assessment for ILC-controlled batch processes in a 2-D system framework. IEEE Trans. Syst. Man Cybern. Syst. 2017, 48, 1493-1504. [CrossRef] 
13. Tian, X.P.; Niu, H.M. A bi-objective model with sequential search algorithm for optimizing network-wide train timetables. Comput. Ind. Eng. 2019, 127, 1259-1272. [CrossRef]

14. Wang, Y.J.; Ding, F.; Xu, L. Some new results of designing an IIR filter with colored noise for signal processing. Digit. Signal Process. 2018, 72, 44-58. [CrossRef]

15. Wong, W.C.; Chee, E.; Li, J.L.; Wang, X.N. Recurrent neural network-based model predictive control for continuous pharmaceutical manufacturing. Mathematics 2018, 6, 242. [CrossRef]

16. Ding, F.; Xu, L.; Alsaadi, F.E.; Hayat, T. Iterative parameter identification for pseudo-linear systems with ARMA noise using the filtering technique. IET Control Theory Appl. 2018, 12, 892-899. [CrossRef]

17. Chen, G.Y.; Gan, M.; Chen, C.L.P.; Li, H.X. A regularized variable projection algorithm for separable nonlinear least squares problems. IEEE Trans. Autom. Control 2019, 64, 526-537. [CrossRef]

18. Li, X.Y.; Li, H.X.; Wu, B.Y. Piecewise reproducing kernel method for linear impulsive delay differential equations with piecewise constant arguments. Appl. Math. Comput. 2019, 349, 304-313. [CrossRef]

19. Xu, L.; Xiong, W.; Alsaedi, A.; Hayat, T. Hierarchical parameter estimation for the frequency response based on the dynamical window data. Int. J. Control Autom. Syst. 2018, 16, 1756-1764. [CrossRef]

20. Zhang, X.; Xu, L.; Ding, F.; Hayat, T. Combined state and parameter estimation for a bilinear state space system with moving average noise. J. Frankl. Inst. 2018, 355, 3079-3103. [CrossRef]

21. Li, M.H.; Liu, X.M.; Ding, F. Filtering-based maximum likelihood gradient iterative estimation algorithm for bilinear systems with autoregressive moving average noise. Circuits Syst. Signal Process. 2018, 37, 5023-5048. [CrossRef]

22. $\mathrm{Xu}, \mathrm{L}$. The damping iterative parameter identification method for dynamical systems based on the sine signal measurement. Signal Process. 2016, 120, 660-667. [CrossRef]

23. Xu, L. A proportional differential control method for a time-delay system using the Taylor expansion approximation. Appl. Math. Comput. 2014, 236, 391-399. [CrossRef]

24. Ding, F.; Chen, H.B.; Xu, L.; Dai, J.Y.; Li, Q.S.; Hayat, T. A hierarchical least squares identification algorithm for Hammerstein nonlinear systems using the key term separation. J. Frankl. Inst. 2018, 355, 3737-3752. [CrossRef]

25. Ding, J.; Chen, J.Z.; Lin, J.X.; Wan, L.J. Particle filtering based parameter estimation for systems with output-error type model structures. J. Frankl. Inst. 2019, 356, 5521-5540. [CrossRef]

26. Liu, N.; Mei, S.; Sun, D.; Shi, W.; Feng, J.; Zhou, Y.M.; Mei, F.; Xu, J.; Jiang, Y.; Cao, X.A. Effects of charge transport materials on blue fluorescent organic light-emitting diodes with a host-dopant system. Micromachines 2019, 10, 344. [CrossRef] [PubMed]

27. Xu, L.; Ding, F. Parameter estimation for control systems based on impulse responses. Int. J. Control Autom. Syst. 2017, 15, 2471-2479. [CrossRef]

28. Cao, Y.; Wang, Z.; Liu, F.; Li, P.; Xie, G. Bio-inspired speed curve optimization and sliding mode tracking control for subway trains. IEEE Trans. Veh. Technol. 2019. [CrossRef]

29. Cao, Y.; Lu, H.; Wen, T. A safety computer system based on multi-sensor data processing. Sensors 2019, 19, 818. [CrossRef]

30. Cao, Y.; Zhang, Y.; Wen, T.; Li, P. Research on dynamic nonlinear input prediction of fault diagnosis based on fractional differential operator equation in high-speed train control system. Chaos 2019, 29, 013130. [CrossRef]

31. Cao, Y.; Li, P.; Zhang, Y. Parallel processing algorithm for railway signal fault diagnosis data based on cloud computing. Future Gener. Comput. Syst. 2018, 88, 279-283. [CrossRef]

32. Wan, L.J.; Ding, F. Decomposition-based gradient iterative identification algorithms for multivariable systems using the multi-innovation theory. Circuits Syst. Signal Process. 2019, 38, 2971-2991. [CrossRef]

33. Chen, J.; Zhu, Q.M.; Li, J.; Liu, Y.J. Biased compensation recursive least squares-based threshold algorithm for time-delay rational models via redundant rule. Nonlinear Dyn. 2018, 91, 797-807. [CrossRef]

34. Yin, C.C.; Wen, Y.Z.; Zhao, Y.X. On the optimal dividend problem for a spectrally positive levy process. Astin Bull. 2014, 44, 635-651. [CrossRef]

35. Yin, C.C.; Wen, Y.Z. Optimal dividend problem with a terminal value for spectrally positive Levy processes. Insur. Math. Econ. 2013, 53, 769-773. [CrossRef]

36. Yin, C.C.; Zhao, J.S. Nonexponential asymptotics for the solutions of renewal equations, with applications. J. Appl. Probab. 2006, 43, 815-824. [CrossRef] 
37. Yin, C.C.; Wang, C.W. The perturbed compound Poisson risk process with investment and debit interest. Methodol. Comput. Appl. Probab. 2010, 12, 391-413. [CrossRef]

38. Yin, C.C.; Wen, Y.Z. Exit problems for jump processes with applications to dividend problems. J. Comput. Appl. Math. 2013, 245, 30-52. [CrossRef]

39. Wen, Y.Z.; Yin, C.C. Solution of Hamilton-Jacobi-Bellman equation in optimal reinsurance strategy under dynamic VaR constraint. J. Funct. Spaces 2019,6750892. [CrossRef]

40. Sha, X.Y.; Xu, Z.S.; Yin, C.C. Elliptical distribution-based weight-determining method for ordered weighted averaging operators. Int. J. Intell. Syst. 2019, 34, 858-877. [CrossRef]

41. Pan, J.; Jiang, X.; Wan, X.K.; Ding, W. A filtering based multi-innovation extended stochastic gradient algorithm for multivariable control systems. Int. J. Control Autom. Syst. 2017, 15, 1189-1197. [CrossRef]

42. Ge, Z.W.; Ding, F.; Xu, L.; Alsaedi, A.; Hayat, T. Gradient-based iterative identification method for multivariate equation-error autoregressive moving average systems using the decomposition technique. J. Frankl. Inst. 2019, 356, 1658-1676. [CrossRef]

43. Ding, F.; Xu, L.; Zhu, Q.M. Performance analysis of the generalised projection identification for time-varying systems. IET Control Theory Appl. 2016, 10, 2506-2514. [CrossRef]

44. $\mathrm{Xu}, \mathrm{L}$; Ding, F. Parameter estimation algorithms for dynamical response signals based on the multi-innovation theory and the hierarchical principle. IET Signal Process. 2017, 11, 228-237. [CrossRef]

45. Zhan, X.S.; Cheng, L.L.; Wu, J.; Yang, Q.S.; Han, T. Optimal modified performance of MIMO networked control systems with multi-parameter constraints. ISA Trans. 2019, 84, 111-117. [CrossRef] [PubMed]

46. Xu, L.; Ding, F.; Zhu, Q.M. Hierarchical Newton and least squares iterative estimation algorithm for dynamic systems by transfer functions based on the impulse responses. Int. J. Syst. Sci. 2019, 50, 141-151. [CrossRef]

47. Wang, F.F.; Ding, F. Partially coupled gradient based iterative identification methods for multivariable output-error moving average systems. Int. J. Model. Identif. Control 2016, 26, 293-302. [CrossRef]

48. Ding, F.; Liu, X.G.; Chu, J. Gradient-based and least-squares-based iterative algorithms for Hammerstein systems using the hierarchical identification principle. IET Control Theory Appl. 2013, 7, 176-184. [CrossRef]

49. Wang, C.; Li, K.C.; Su, S. Hierarchical Newton iterative parameter estimation of a class of input nonlinear systems based on the key term separation principle. Complexity 2018, 2018, 7234147. [CrossRef]

50. Ding, F.; Liu, G.; Liu, X.P. Partially coupled stochastic gradient identification methods for non-uniformly sampled systems. IEEE Trans. Autom. Control 2010, 55, 1976-1981. [CrossRef]

51. Liu, Q.Y.; Ding, F.; Yang, E.F. Parameter estimation algorithm for multivariable controlled autoregressive autoregressive moving average systems. Digit. Signal Process. 2018, 83, 323-331. [CrossRef]

52. Liu, Q.Y.; Ding, F.; Xu, L.; Yang, E.F. Partially coupled gradient estimation algorithm for multivariable equation-error autoregressive moving average systems using the data filtering technique. IET Control Theory Appl. 2019, 13, 642-650. [CrossRef]

53. Ding, F. Coupled-least-squares identification for multivariable systems. IET Control Theory Appl. 2013, 7, 68-79. [CrossRef]

54. Ding, F. Two-stage least squares based iterative estimation algorithm for CARARMA system modeling. Appl. Math. Model. 2013, 37, 4798-4808. [CrossRef]

55. $\mathrm{Xu}, \mathrm{L}$. Application of the Newton iteration algorithm to the parameter estimation for dynamical systems. J. Comput. Appl. Math. 2015, 288, 33-43. [CrossRef]

56. Ding, F.; Meng, D.D.; Dai, J.Y.; Li, Q.S.; Alsaedi, A.; Hayat, T. Least squares based iterative parameter estimation algorithm for stochastic dynamical systems with ARMA noise using the model equivalence. Int. J. Control Autom. Syst. 2018, 16, 630-639. [CrossRef]

57. Wang, Y.J.; Ding, F. Iterative estimation for a non-linear IIR filter with moving average noise by means of the data filtering technique. IMA J. Math. Control Inf. 2017, 34, 745-764. [CrossRef]

58. Li, M.H.; Liu, X.M. The least squares based iterative algorithms for parameter estimation of a bilinear system with autoregressive noise using the data filtering technique. Signal Process. 2018, 147, 23-34. [CrossRef]

59. Ding, F.; Pan, J.; Alsaedi, A.; Hayat, T. Gradient-based iterative parameter estimation algorithms for dynamical systems from observation data. Mathematics 2019, 7, 428. [CrossRef]

60. Ding, F.; Wang, Y.J.; Dai, J.Y.; Li, Q.S.; Chen, Q.J. A recursive least squares parameter estimation algorithm for output nonlinear autoregressive systems using the input-output data filtering. J. Frankl. Inst. 2017, 354, 6938-6955. [CrossRef] 
61. Zhang, X.; Ding, F.; Alsaadi, F.E.; Hayat, T. Recursive parameter identification of the dynamical models for bilinear state space systems. Nonlinear Dyn. 2017, 89, 2415-2429. [CrossRef]

62. Xu, L.; Ding, F. Recursive least squares and multi-innovation stochastic gradient parameter estimation methods for signal modeling. Circuits Syst. Signal Process. 2017, 36, 1735-1753 [CrossRef]

63. Zhang, X.; Ding, F.; Xu, L.; Alsaedi, A.; Hayat, T. A hierarchical approach for joint parameter and state estimation of a bilinear system with autoregressive noise. Mathematics 2019, 7, 356. [CrossRef]

64. Liu, Q.Y.; Ding, F. Auxiliary model-based recursive generalized least squares algorithm for multivariate output-error autoregressive systems using the data filtering. Circuits Syst. Signal Process. 2019, 38, 590-610. [CrossRef]

65. Ding, F. Decomposition based fast least squares algorithm for output error systems. Signal Process. 2013, 93, 1235-1242. [CrossRef]

66. Ding, F.; Liu, X.P.; Liu, G. Gradient based and least-squares based iterative identification methods for OE and OEMA systems. Digital Signal Process. 2010, 20, 664-677. [CrossRef]

67. Wang, Y.J.; Ding, F.; Wu, M.H. Recursive parameter estimation algorithm for multivariate output- error systems. J. Frankl. Inst. 2018, 355, 5163-5181. [CrossRef]

68. Ding, J.L. The hierarchical iterative identification algorithm for multi-input-output-error systems with autoregressive noise. Complexity 2017, 2017, 5292894. [CrossRef]

69. Wang, X.H.; Ding, F. Partially coupled extended stochastic gradient algorithm for nonlinear multivariable output error moving average systems. Eng. Comput. 2017, 34, 629-647. [CrossRef]

70. Zhang, X.; Ding, F.; Xu, L.; Yang, E.F. State filtering-based least squares parameter estimation for bilinear systems using the hierarchical identification principle. IET Control Theory Appl. 2018, 12, 1704-1713. [CrossRef]

71. Huang, W.; Ding, F.; Hayat, T.; Alsaedi, A. Coupled stochastic gradient identification algorithms for multivariate output-error systems using the auxiliary model. Int. J. Control Autom. Syst. 2017, 15, 1622-1631. [CrossRef]

72. Wang, Y.J.; Ding, F. The filtering based iterative identification for multivariable systems. IET Control Theory Appl. 2016, 10, 894-902. [CrossRef]

73. Meng, D.D. Recursive least squares and multi-innovation gradient estimation algorithms for bilinear stochastic systems. Circuits Syst. Signal Process 2016, 35, 1052-1065. [CrossRef]

74. Wan, X.K.; Li, Y.; Xia, C.; Wu, M.H.; Liang, J.; Wang, N. A T-wave alternans assessment method based on least squares curve fitting technique. Measurement 2016, 86, 93-100. [CrossRef]

75. Zhao, N. Joint Optimization of cooperative spectrum sensing and resource allocation in multi-channel cognitive radio sensor networks. Circuits Syst. Signal Process. 2016, 35, 2563-2583. [CrossRef]

76. Zhao, X.L.; Liu, F.; Fu, B.; Na, F. Reliability analysis of hybrid multi-carrier energy systems based on entropy-based Markov model. Proc. Inst. Mech. Eng. Part O-J. Risk Reliab. 2016, 230, 561-569. [CrossRef]

77. Zhao, N.; Liang, Y.; Pei, Y. Dynamic contract incentive mechanism for cooperative wireless networks. IEEE Trans. Veh. Technol. 2018, 67, 10970-10982. [CrossRef]

78. Gong, P.C.; Wang, W.Q.; Li, F.C.; Cheung, H. Sparsity-aware transmit beamspace design for FDA-MIMO radar. Signal Process. 2018, 144, 99-103. [CrossRef]

79. Zhao, X,L.; Lin, Z.Y.; Fu, B.; He, L.; Na, F. Research on automatic generation control with wind power participation based on predictive optimal 2-degree-of-freedom PID strategy for multi-area interconnected power system. Energies 2018, 11, 3325. [CrossRef]

80. Liu, F.; Xue, Q.; Yabuta, K. Boundedness and continuity of maximal singular integrals and maximal functions on Triebel-Lizorkin spaces. Sci. China Math. 2019. [CrossRef]

81. Liu, F. Boundedness and continuity of maximal operators associated to polynomial compound curves on Triebel-Lizorkin spaces. Math. Inequal. Appl. 2019, 22, 25-44. [CrossRef]

82. Liu, F.; Fu, Z.; Jhang, S. Boundedness and continuity of Marcinkiewicz integrals associated to homogeneous mappings on Triebel-Lizorkin spaces. Front. Math. China 2019, 14, 95-122. [CrossRef]

83. Wang, D.Q.; Yan, Y.R.; Liu, Y.J.; Ding, J.H. Model recovery for Hammerstein systems using the hierarchical orthogonal matching pursuit method. J. Comput. Appl. Math. 2019, 345, 135-145. [CrossRef]

84. Zhang, S.; Wang, D.Q.; Liu, F. Separate block-based parameter estimation method for Hammerstein systems. R. Soc. Open Sci. 2018, 5, 172194. [CrossRef] [PubMed] 
85. Wang, D.Q.; Zhang, Z.; Xue, B.Q. Decoupled parameter estimation methods for Hammerstein systems by using filtering technique. IEEE Access 2018, 6, 66612-66620. [CrossRef]

86. Wang, D.Q.; Li, L.W.; Ji, Y.; Yan, Y.R. Model recovery for Hammerstein systems using the auxiliary model based orthogonal matching pursuit method. Appl. Math. Modell. 2018, 54, 537-550. [CrossRef]

87. Feng, L.; Li, Q.X.; Li, Y.F. Imaging with 3-D aperture synthesis radiometers. IEEE Trans. Geosci. Remote Sens. 2019, 57, 2395-2406. [CrossRef]

88. Shi, W.X.; Liu, N.; Zhou, Y.M.; Cao, X.A. Effects of postannealing on the characteristics and reliability of polyfluorene organic light-emitting diodes. IEEE Trans. Electron Devices 2019, 66, 1057-1062. [CrossRef]

89. Fu, B.; Ouyang, C.X.; Li, C.S.; Wang, J.W.; Gul, E. An improved mixed integer linear programming approach based on symmetry diminishing for unit commitment of hybrid power system. Energies 2019, 12, 833. [CrossRef]

90. Wu, T.Z.; Shi, X.; Liao, L; Zhou, C.J.; Zhou, H.; Su, Y.H. A capacity configuration control strategy to alleviate power fluctuation of hybrid energy storage system based on improved particle swarm optimization. Energies 2019, 12, 642. [CrossRef]

91. Zhao, N.; Chen, Y.; Liu, R.; Wu, M.H.; Xiong, W. Monitoring strategy for relay incentive mechanism in cooperative communication networks. Comput. Electr. Eng. 2017, 60, 14-29. [CrossRef]

92. Zhao, N.; Wu, M.H.; Chen, J.J. Android-based mobile educational platform for speech signal processing. Int. J. Electr. Eng. Educ. 2107, 54, 3-16. [CrossRef]

93. Wan, X.K.; Wu, H.; Qiao, F.; Li, F.; Li, Y.; Wan, Y.; Wei, J. Electrocardiogram baseline wander suppression based on the combination of morphological and wavelet transformation based filtering. Comput. Math. Methods Med. 2019, 7196156. [CrossRef] [PubMed]

94. Ma, F.Y.; Yin, Y.K.; Li, M. Start-up process modelling of sediment microbial fuel cells based on data driven. Math. Probl. Eng. 2019, 7403732. [CrossRef]

95. Yang, F.; Zhang, P.; Li, X.X. The truncation method for the Cauchy problem of the inhomogeneous Helmholtz equation. Appl. Anal. 2019, 98, 991-1004. [CrossRef]

96. Sun, Z.Y.; Zhang, D.; Meng, Q.; Cheng, C.C. Feedback stabilization of time-delay nonlinear systems with continuous time-varying output function. Int. J. Syst. Sci. 2019, 50, 244-255. [CrossRef]

97. Wu, M.H.; Li, X.; Liu, C.; Liu, M.; Zhao, N.; Wang, J.; Wan, X.K.; Rao, Z.H.; Zhu, L. Robust global motion estimation for video security based on improved k-means clustering. J. Ambient Intell. Hum. Comput. 2019, 10, 439-448. [CrossRef]

98. Zhao, X.L.; Lin, Z.Y.; Fu, B.; He, L.; Li, C.S. Research on the predictive optimal PID plus second order derivative method for AGC of power system with high penetration of photovoltaic and wind power. J. Electr. Eng. Technol. 2019, 14, 1075-1086. [CrossRef]

(C) 2019 by the authors. Licensee MDPI, Basel, Switzerland. This article is an open access article distributed under the terms and conditions of the Creative Commons Attribution (CC BY) license (http://creativecommons.org/licenses/by/4.0/). 